\title{
Dust and Stellar Populations in the Large Magellanic Cloud
}

\author{
Dennis Zaritsky ${ }^{1}$ \\ UCO/Lick Observatories and Board of Astronomy and Astrophysics, \\ Univ. of California at Santa Cruz, Santa Cruz, CA, 95064 \\ dennis@ucolick.org
}

\begin{abstract}
We present an analysis of line-of-sight extinction measurements obtained using data from the Magellanic Clouds Photometric Survey (Zaritsky, Harris, \& Thompson 1997, AJ, 114, 1002), which provides 4-filter photometry for millions of stars in the Large Magellanic Cloud. We find that visual extinctions are typically larger by several tenths of a magnitude for stars with effective temperatures $>12000 \mathrm{~K}$, than for stars with effective temperatures between $5500 \mathrm{~K}$ and 6500 K. Several repercussions of this population-dependent extinction are discussed. In particular, LMC distance measurements that utilize old stellar populations, but use extinctions derived from OB stars, may be biased low. As a specific example, we show that the LMC distance modulus derived from field red clump stars is revised upward relative to published measurements by $\sim 0.2 \mathrm{mag}$ if one uses the extinction measured for a matched stellar population. Conversely, measurements that utilize the youngest stars are subject to greater, and more variable, extinction leading preferentially to results that may be biased high. Population-dependent extinction affects the interpretation of color-magnitude diagrams and results in an effective absorption law that is steeper than that intrinsic to the dust for unresolved stellar systems. We further explore the relation between the stellar populations and dust by comparing our extinction map to the $100 \mu \mathrm{m}$ image of the region and identifying potential heating sources of the dust. We find that although regions of high $100 \mu \mathrm{m}$ flux are associated with young stars, young stars are not necessarily associated with regions of high $100 \mu \mathrm{m}$ flux and that $\sim 50 \%$ of the $100 \mu \mathrm{m}$ flux is emitted beyond the immediate regions of high OB stellar density. We conclude that $100 \mu \mathrm{m}$ flux should be used with caution as a star formation tracer, particularly for studies of star formation within galaxies. Finally, we reproduce the observed extinction variation between the hot and cold stellar populations with a simple model of the distribution of the stars and dust where the scaleheight of the cooler stars is $\gg$ than that of the dust (which is twice that of the OB stars; Harris, Zaritsky, \& Thompson 1997, AJ, 114, 1933).
\end{abstract}

Subject headings: dust, extinction — galaxies: distances and redshifts — galaxies: photometry — Magellanic Clouds

\section{Introduction}

The distribution of dust within galaxies and its effect on our observations are poorly determined and have serious implications for many areas of extragalactic astronomy. In a previous paper (Harris, Zaritsky, \& Thompson 1997; hereafter

\footnotetext{
${ }^{1}$ Present Address: Steward Observatory, Univ. of Arizona, Tucson, AZ, 85721
}

HZT), we applied the classic technique of determining line-of-sight extinction from the reddeningfree colors of OB stars (Johnson \& Morgan 1953) to produce an extinction map of a $1.9^{\circ} \times 1.5^{\circ}$ region of the Large Magellanic Cloud (LMC) and to investigate the relative line-of-sight distributions of the dust and OB stars. We found that the OB stars in the LMC have a scaleheight that is about half that of the dust. The combination of this result and the observed spatial variations among 
different stellar populations (cf. Harris and Zaritsky 1999) suggests that each stellar population will have its own distinct distribution of extinction values. Can the relative distributions of stellar populations and dust be mapped and what effects does this variation have on observations of the Large Magellanic Cloud in particular and of galaxies in general?

The correlation between dust and certain stellar populations is well-known (e.g., OB stars and dust are preferentially found in galactic spiral arms), but generally ignored when treating the global effect of dust on galaxy photometry due to the complexity and ambiguity such a treatment introduces. One specific example where such a correlation may be important is found in the measurement of the distance to the Magellanic Clouds. Different reddenings toward Cepheid, RR Lyrae, RGB tip stars, and red clump stars would result in apparent differences among the derived distance moduli for each of these populations. Apparent differences of a few tenths of a magnitude in distance moduli are observed (eg. $m-M \sim 18.3$ for RR Lyrae and red clump stars; Layden et al. 1996; Udalski et al. 1998; Stanek et al. 1998; Cole 1998, Girardi et al. 1998; and $m-M \sim 18.5$ to 18.7 for Cepheids; Madore and Freedman (1998) and Feast and Catchpole (1997)) and render the distance measurements to this key calibration galaxy systematically uncertain to about $10 \%$. Although there are many factors that give rise to the discrepancies, the differences, at least in part, may arise from applying extinctions derived from a different stellar populations than that being used as the standard candle.

For unresolved galaxies, even the most detailed reddening corrections are fairly rudimentary (typically assuming uniform, symmetric distributions of dust; cf. Tully \& Foqué 1985). Although increasingly more detailed treatments are being constructed and applied (Huizinga 1995; Witt \& Gordon 1996; Jones, Davies, \& Trewhella 1996; Kuchinski et al. 1998), these focus primarily on the effect of a clumpy absorbing media and have not yet included the additional complexity of varied distributions of dust and stellar populations. A complete treatment is obviously a difficult, complicated problem for which observed examples of dust/star geometries would provide invaluable constraints. The Clouds provide an opportunity to measure the effects of a non-uniform dust distribution on different stellar populations through line-of-sight extinction measurements for millions of stars.

Once the distributions of stars and dust are determined, we can begin to investigate issues regarding detailed physics of the interstellar medium. A specific example that we can address is the outstanding issue of how much of the far infrared emission comes from dust heated by the UV flux from nearby OB stars (Young et al. 1986; Devereux \& Young 1990) rather than by the galactic interstellar radiation field (Lonsdale-Persson \& Helou 1987, Walterbos \& Schwering 1987). The resolution of this question impacts the degree to which the far-IR flux can be used to measure, or at least trace, recent star formation activity. At least two dust components are acknowledged to be contributing to the far infrared flux: a warm component associated with H II regions (and hence massive stars) and a cold component, the cirrus, that is heated by the interstellar radiation field. Previous studies have by necessity focused on galaxies with marginally resolved stellar populations (e.g., M33; Devereux, Duric, \& Scowen 1997 ) or unresolved populations (cf. Young et al. 1996; Lonsdale-Persson \& Helou 1987), so it has been difficult to investigate the detailed physical connection between stars and dust emission. The contribution from the cirrus component is thought to depend on Hubble type (Sauvage \& Thuan 1992) decreasing to a contribution of $\sim 3 \%$ in Sdm galaxies, like the LMC. The expectation that little $100 \mu \mathrm{m}$ emission will be unassociated with young stars in the LMC is simple to test because regions populated by young stars are easily identifiable from our photometry, the IRAS image has resolution comparable to the sizes of star forming regions, and our extinction map provides a measurement of the dust column density.

With line-of-sight extinction measurements to a wide set of stellar populations within the LMC, we can begin to explore the relative distribution of dust and stellar populations, at least in this one galaxy. This study is a necessary step to the accurate detailed interpretation of the rich colormagnitude diagrams of the Clouds currently being produced. To motivate this study in a wider context, we have described a few ways in which a dust map of the LMC relative to various stel- 
lar populations may address issues in extragalactic astronomy and ISM physics. The previous study of extinction in the LMC with the highest spatial resolution utilized $2069 \mathrm{O}$ and $\mathrm{B}$ main sequence stars drawn from a dataset of over 1 million stars (HZT). To extend the range of stellar types included in the extinction analysis and enlarge the sample, we develop and describe a technique that measures the extinction by fitting stellar models to the stellar spectral energy distribution. We derive estimates of both the reddening and effective temperature of every star in our survey for which 4-band photometry is available. In Section 2 we briefly describe the data from a region of about $4^{\circ} \times 2.7^{\circ}$ in the LMC. In Section 3 we describe our analysis and in Section 4 our results.

\section{Data}

These data come from the ongoing Magellanic Clouds Photometric Survey described initially by Zaritsky, Harris, \& Thompson (1997; hereafter ZHT) which is a $U B V I$ imaging survey conducted with the Las Campanas Swope (1m) telescope, the Great Circle Camera (Zaritsky, Shectman, \& Bredthauer 1996), and a $2048 \times 2048$ pixel CCD with pixel scale of 0.7 arcsec pixel ${ }^{-1}$. The data reduction is described in the original paper except for one modification.

We revise the photometric registration algorithm because the results were unsatisfactory when the original scheme was applied to the 14 drift scans included here. We developed a superior alternate algorithm based on adjusting the photometry of the individual subscans reduced with DAOPHOT II (Stetson 1987). We assume that the photometry of each subscan can be related to the true photometry by a single zero point offset, and we assume that on average the photometry from the survey is accurate (i.e. has a net zero point of $0.0 \mathrm{mag})$. We calculate the relative zero point for a particular subscan by evaluating the median of the photometric shifts relative to all of the neighboring subscans (calculated using stars in common within the overlap regions between adjacent subscans). Cycling through all of the subscans, we identify the subscan with the largest median offset. Its photometry is adjusted by that offset, and the algorithm begins again. We stop iterating when the the largest subscan offset is less than $0.02 \mathrm{mag}$ (a value that we judge to be below the noise of this procedure). The principal remaining difficulty is an artifact of the peculiar subset of data currently available. The presence of a gap in coverage down the middle of the region (cf. Figure 1) renders the relative zero points of the right and left half of the region less than ideal (and possible issues raised by this deficiency will be discussed). The gap will be filled as the survey progresses and the entire region described in this paper will be surrounded by adjacent scans.

\section{Measuring Extinction}

To measure the extinction we fit three parameters, the effective temperature, the luminosity, and the extinction, to the four-filter photometry of each star. Because we only have four data values to fit per star, more sophisticated modeling (eg. an exploration of the extinction curve) is unwarranted. The requirement that a star be detected in all four bands eliminates a substantial fraction (0.60) of the entire sample of stars, because the $U$ and $I$ band images are shallower than the $B$ and $V$ images. Even though the procedure appears relatively straightforward there are various subtleties that must be addressed.

First, we must adopt a set of suitable stellar models to fit. We use the library of models provided by Lejeunne, Cuisinier, and Buser (1997). These models span a wide range of metallicities, effective temperature $\left(T_{E}\right.$, and loosely referred to as the temperature throughout this paper), and $\log g$. Special care was taken by those authors to place the models on the correct color scale so that the theoretical solar-abundance model yields the observed solar $U$ through $L$ colors. This correction is particularly important for a use such as that being described here. Of their many libraries, we use the solar metallicity library (lcbp00.cor) for calibration and for a test of the metallicity dependence of the method, the $[\mathrm{m} / \mathrm{H}]=-0.5$ library (lcbm05.cor) for the LMC analysis (the average $[\mathrm{O} / \mathrm{H}]$ gas phase abundance among LMC HII regions is -0.53 ; Pagel et al. 1978), and the $[\mathrm{m} / \mathrm{H}]$ $=-1$ library (lmbm10.cor) for a test of the metallicity dependence of the algorithm.

Second, we must adopt a conversion system between magnitudes and fluxes. One option is to adopt the absolute flux densities of an A0V star 
from an existing reference, such as Straižys (1992). In principle, the standard star's flux densities enable us to convert any flux into a magnitude, or vice versa. However, our photometric system may not be an exact match to this particular photometric system or to that adopted for the theoretical models. Instead, we adopt a more self-consistent approach by defining one of the models to represent an A0V star $\left(T_{E}=9500^{\circ}\right.$ and $\left.\log (g)=4\right)$. We calculate fluxes through the our filter system and assign these fluxes to a 0 mag star in each filter. With this calibration, fluxes and magnitudes through each of the four passbands can be evaluated for any temperature-surface gravity model.

Third, we must adopt a function for the wavelength dependence of extinction across the optical portion of the spectrum. Our adopted extinction law is a standard Galactic curve (Schild 1977 and references therein), which for $\lambda>2600 \AA$ is virtually identical to the LMC extinction curve even in environments that show significant differences

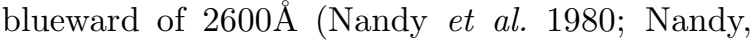
Morgan, \& Houziaux 1984). We parameterize the normalization of the extinction law using the extinction in the visual band, $A_{V}$. Variations in $R_{V}$, $A_{V} / E(B-V)$, are known to occur in the galaxy (cf. Carrasco, Strom, \& Strom 1973; Cardelli, Clayton, \& Mathis 1989), but we cannot resolve such variations in the LMC with our data. Koornneef (1982) demonstrated that the typical value of $R_{V}$ for the LMC is similar to that of the typical line of sight in the Galaxy, 3.1 (which is the adopted value for this study).

The temperature and line-of-sight extinction of a particular star are then derived by identifying the best fit model for that star. The algorithm compares the observed flux in each passband to that calculated from a model. The model is renormalized to minimize residuals and $\chi^{2}$ is calculated using the observational uncertainties. We allow the flux to be renormalized to avoid assuming a distance to the star (which is a more critical free parameter for the local calibrating sample to be discussed than for the LMC stars). The residuals are calculated as fractional differences between the observed and predicted flux (i.e. $\Delta$ mag, which weighs each filter in the determination of $\chi^{2}$ more equally than if $\Delta$ flux is used). We cycle through all available stellar models with $T>3000 \mathrm{~K}$ and for each model, we cycle through $0 \leq A_{V} \leq 2$ in steps of $0.01 \mathrm{mag}$. The parameters of the model with the lowest value of $\chi^{2}$ are accepted as the stellar temperature and $A_{V}$.

We use local stars of known spectral type and magnitude to calibrate and test the procedure. Stellar data, $U B V I$ magnitudes and spectral types, are collected from a series of catalogs available on-line at the Strasbourg data center (http://cdsarc.u-strasbg.fr/; Lanz 1986; Mermilliod 1986; Houk \& Smith-Moore 1988) for 107 HD stars. Using the relationship between stellar type and effective temperature given by Straižys (1982), we convert the spectral type into an effective temperature for each star. In Figure 2 we show the results from various tests of fitting the stellar models to these data. In the upper left panel of the Figure, we have plotted the derived effective temperatures versus the known temperatures, which we refer to as spectroscopic temperatures, obtained by assuming zero extinction along the line of sight and no photometric offset between the theoretical photometric system of the models plus our filter transmission curves and that used to produce the published magnitudes.

The agreement between the two temperatures is encouraging but there are two qualitative failures that must be corrected. First, the derived temperatures are systematically lower than the spectroscopic temperatures. Second, the deviations become large for stars with $T_{E}>10000 \mathrm{~K}$. In the upper right panel, we plot the results allowing the algorithm to fit extinctions. The agreement is significantly better. There is no noticeable offset between photometric and spectroscopic temperatures for stars with $T_{E}<10000 \mathrm{~K}$ and the situation has improved somewhat for the hotter stars as well. Next we allow the photometric zero points of each filter to change, and we select the best offsets by minimizing the residuals about the 1:1 line. The results obtained with these offsets are shown in the lower left panel. We have improved the fit at the hotter effective temperatures, although this improvement has been obtained by construction. The photometric offsets are modest (1\% in $U$ and $B, 7 \%$ in $V$ and $12 \%$ in $I$ ). These offsets may in part arise from a mismatch of the reference model and an A0V star or from differences in the filter transmission curves between photometric systems. Finally in the last panel (lower right), we plot the result of applying the algorithm (with freedom to 
fit extinction and with the new photometric offsets) to data for $U B V R I$ standards taken from the Astronomical Almanac (1981). Again, we have converted from stellar types to $T_{E}$ using the conversion table from Straižys (1982). The photometric temperature agrees well with the spectroscopic temperature over the range $3000 \mathrm{~K}$ to $35000 \mathrm{~K}$. The agreement between the fitted and published temperatures for this independent sample demonstrates that the photometric offsets derived using the first sample are generally appropriate for other data as well. We conclude that we can recover $T_{E}$ to a fractional precision of $\sim 10 \%$ over this temperature range.

Although we obtain estimates of the line-ofsight extinction toward these standard stars, there are no published measurements to compare with. Instead, we test our extinction measurements by artificially reddening the photometry of these stars and attempting to recover the added extinction. This approach tests the degeneracy among various stellar models plus reddening, but does not test the extinction law itself. We apply the same supplemental visual extinction $(0.2,0.4$, and 0.8 mag) to the 107 standard stars, refit the models, and accept the best fits only if $\chi^{2}<3$ (as we will do with the analysis of LMC stars). In Figure 3 we plot a running median of the newly derived extinction minus the initial derived extinction (bins of 11 stars, with stars sorted by photometric temperature) and compare the result with the input supplemental extinction, which is plotted as a dotted line. For low supplemental extinction values, $A_{V}=0.2$, which corresponds to low total extinction because the initial extinctions are generally small, the supplemental extinction is recovered well across the entire range of temperatures. As the extinction increases, we notice some serious regions of failure. In particular, the region of photometric $T_{E}$ between 7000 and $9000 \mathrm{~K}$ has extinction values that are significantly underestimated for supplemental extinctions of 0.4 and 0.8 mag.

To examine the failure of the method for stars of intermediate temperatures, we plot the results for supplemental extinction of 0.8 , but sort stars by spectroscopic, rather than photometric, temperature (lowest panel of Figure 3). By comparing the bottom two panels, in particular the temperature range over which the $A_{V}$ determination fails, we can understand what has happened. Stars with large extinctions and $T_{E} \sim 10000 \mathrm{~K}$ are well fit by models with cooler stellar atmospheres and smaller $A_{V}$. This problem is a manifestation of the classic degeneracy evident when applying the reddening-free method in the $U-B, B-V$ colorcolor diagram. For small extinctions, the degeneracy is avoided, but for large extinctions various combinations of $T_{E}$ and $A_{V}$ are possible for stars with true $T_{E}$ 's between 7000 and $12000 \mathrm{~K}$. We also see a failure mode below $5000 \mathrm{~K}$ for our algorithm. We suspect that the problem at these low temperatures is a combination of insensitivity to reddening in stars that are already quite red and the effect of strong molecular absorption features that effectively introduce noise into the process of matching the models to the observations. We conclude that for the range of extinctions expected in the LMC, we should constrain our analysis to stars with photometrically derived $T_{E}$ 's between 5500 and 6500 $\mathrm{K}$ and greater than $12000 \mathrm{~K}$.

We run the algorithm on the stellar catalog obtained for our survey region using the $[\mathrm{m} / \mathrm{H}]$ $=-0.5$ models and the adopted photometric offsets. We only use stars with moderate or better photometric errors $\left(\sigma_{U} \leq 0.2, \sigma_{B} \leq 0.1, \sigma_{V} \leq 0.1\right.$, and $\sigma_{I} \leq 0.1$ ) and apply magnitude cuts (exclude stars with $U$ and $B>22$ and $V$ and $I>21)$. We obtain satisfactory fits $\left(\chi^{2}<3\right)$ for $94 \%$ of the stars that satisfy our criteria (a total of 543,860 stars within this region). For our subsequent analysis we exclude stars with fitted $T_{E}<5500 \mathrm{~K}$ and $6500 \mathrm{~K}<T_{E}<12000 \mathrm{~K}$. In the lower allowed temperature range we have 39,613 stars and in the upper allowed temperature range we have 106,181 stars.

The various photometric selection criteria pose a potential risk of introducing unwanted biases. A limiting magnitude necessarily implies that heavily extincted stars will be lost from the sample. The selection based on photometric errors similarly introduces a "fuzzy" magnitude limit because the errors correlate strongly with magnitude. Except for extremely extincted stars, these limits are unlikely to be important for the hotter stars because those stars are at least a magnitude brighter than the $V$ band limit and have $B-V \sim 0$. There is greater potential for biases in the colder sample because these stars are fainter and redder, and so more likely to be lost from the $U$ band data, 
which is our shallowest. Upon inspection we find that although the magnitude limit $(U>22)$ is well below ( $\sim 2 \mathrm{mag}$ ) the red clump population, which is where the bulk of the cold stars lie, the photometric error cut effectively raises the limiting magnitude to $\sim 21 \mathrm{mag}$. To test whether this seriously affects the recovered distribution of extinctions we have selected a second sample of cold stars with $0.2<\sigma_{U}<0.4$ to recover some of the excluded stars. The comparison of extinction values among the two samples is presented in Figure 4. There is no indication that high extinction $(>0.4)$ stars are being artificially dropped from the sample due to the $\sigma_{U}<0.2$ cut. We conclude that although there is no way to avoid losing the fainter stars along lines-of-sight with extreme extinctions $(>1$ mag), selection criteria are not significantly biasing the recovered distribution of $A_{V}$ 's.

Using the derived line-of-sight extinction values, we construct an extinction map by spatially grouping the measurements. We regrid the survey region and begin with a map pixel that corresponds to 120 original pixels (84 arcsec). Regions of high stellar density could be treated with smaller map pixels, but this choice of pixel size was found to be a good compromise for the entire survey region. For map pixels that contain at least three stars with extinction measurements the median extinction value is adopted. For map pixels with two or fewer values, we increase the size of the map pixel size by another 120 original pixels. If fewer than three stars are found within this larger map pixel, we increase the map pixel size and try again. We continue incrementing the map pixel size until the new, larger pixel contains at least three extinction values. All original pixels contained within the enlarged map pixels are assigned the median value. This algorithm results in a final map that has regions of high and low spatial resolution, but relatively uniform signalto-noise. Finally, we create a mask that sets any original map pixel containing zero usable stars to zero extinction because such pixels are likely to be either off the surveyed region or to be corrupted by technical problems.

The two maps resulting from the analysis of high and low $T_{E}$ stars are presented in Figure 5 (with higher extinction regions shaded darker). It is evident that the average extinction values are larger for the hotter stars. We will return to this point in Section 4. We have marked, with a vertical line to the right of the images, the scan that is known to have $I$-band data taken in nonphotometric conditions and we suspect that the feature at the far right of that scan (near the right edge) is a result of these non-photometric data. These non-photometric data, through the process of photometric registration discussed in $\S 2$, may also have partially corrupted the data adjacent to the top of the non-photometric scan, which appear to have an anomalously low $A_{V}$ in the upper panel of the Figure. Finally, the other feature we note in our preliminary discussion of the maps is the left/right offset present in the lower panel. We cautioned previously that the central coverage gap renders the relative photometric normalization of the left and right sections somewhat uncertain (0.05 mag) and a slight shift in the mean extinction is seen between the two sides for the cool stars. Other than these issues, there is some slight structure that correlates with subscan or scan edges.

\section{Discussion}

The extinction maps shown in Figure 5, in particular the one derived from the stars with $T_{E}>12000 \mathrm{~K}$, show large coherent, filamentary structures. Most noticeable is the broken ring of extinction in the left hand of the survey region that surrounds the central stellar association of LMC-4. Most of the high extinction regions visible in the top panel of Figure 5 can also be seen as regions of obscuration in the stellar density map (Figure $1)$. This coincidence confirms the features in the extinction map as real regions of enhanced extinction. Because of the larger number of stars used, we can trace features at finer spatial resolution than done by HZT. After examining the sensitivity of the algorithm to the choice of metallicity, we proceed by comparing the reddening map to previous reddening maps and other observations of the same region.

\subsection{Variation with Metallicity}

Although the difference in the inferred extinction values between high and low $T_{E}$ stars is significant, we must exclude the possibility that this difference is due to physical reasons other than a difference in line-of-sight extinction. One such 
possible reason is the metallicity difference that presumably exists between the younger and older stellar populations. We have selected to fit models that correspond to the current gas-phase abundance in the LMC for all of the stars, which may not be appropriate for the low $T_{E}$ stars. We test the technique's sensitivity to mismatched metallicities between the stars and models by reapplying the algorithm using tenth-solar and solar metallicity models on a random subset of the data.

The resulting differences in $T_{E}$ and $A_{V}$ as a function of $T_{E}$ are shown in Figure 6 (we plot the difference in the inferred quantity between a run using the standard $[\mathrm{m} / \mathrm{H}]=-0.5$ models and the other $[\mathrm{m} / \mathrm{H}]$ models). There are no systematic differences in either the effective temperature or extinction, except possibly at temperatures below $5000 \mathrm{~K}$ for $A_{V}$ when using the solar metallicity models. In both plots of the derived extinction differences, there are one or more points, within our $T_{E}$ range, for which the average difference is large ( $\gtrsim 0.1 \mathrm{mag})$. For those points, we have also evaluated the median value within those bins and plotted these values as open circles. The median, which we use when generating our extinction maps, is consistent with zero difference. A change of a factor of 10 in the adopted abundance appears to have little or no net effect on the derivation of $A_{V}$ and $T_{E}$ over large samples of stars. This result reflects some of the difficulty in obtaining metallicity measurements for individual stars from broad band colors, and assures us that metallicity mismatches, which are certainly present at some level, are not responsible for the offset seen in $A_{V}$ between high and low $T_{E}$ stars.

\subsection{Comparison to HZT's OB Star Ex- tinction Measurements}

A straightforward test of the derived extinction values for the high $T_{E}$ stars is a direct comparison with previous results. HZT compared their results to previous studies (primarily, Hill et al. 1994, Massey et al. 1995, Oestreicher \& SchmidtKaler 1996) and found that if similar selection criteria are used the distribution of reddening values agree. Because the HZT results are based on a subset of the data used here, the comparison to the HZT results that we describe below directly tests our algorithm. The agreement between the HZT results and previous studies address the reli- ability of the data.

We match the 1615 stars in common between the two studies and present the comparison in Figure 7 . On average the two measurements agree well and there is no indication of a bias between the two techniques, but the dispersion about the 1:1 line is large, $0.21 \mathrm{mag}$. Some of this scatter is attributable to the different photometric zero pointing technique used here and correlates with position in the survey region. The scatter among extinction measurements within smaller areas of the survey is about half of the global scatter and this reduced local scatter gives rise to the appearance of two tighter sequences of stars above and below the 1:1 line. From this comparison, we conclude that the uncertainty in $A_{V}$ for a single star is at least as large as $0.1 \mathrm{mag}$ and possibly as large as $0.2 \mathrm{mag}$. The comparison demonstrates that the reddening to any individual star is significantly uncertain and the results from these techniques must be averaged over several stars, as we do to produce our extinction maps, to mitigate these uncertainties. Nevertheless, we believe that the newer photometric zero-pointing technique is superior and so the current measures of $A_{V}$ are preferable over the HZT values. For the averaging process to be physically meaningful, the region over which the averaging is done must have homogeneous extinction properties and the final extinction map will not resolve structures on scales smaller than the size of the region chosen for averaging or medianing.

\subsection{Comparison to Published Extinctions for OB Regions}

We compare our reddening values with those published for $\mathrm{OB}$ regions within our survey. We have identified four Lucke-Hodge regions ((LH 38, 54, 83, and 114; Lucke and Hodge 1970) in common between our survey and the studies by Hill, Madore, and Freedman (1994) and Oey (1996). Our median values of $E(B-V)$ for stars in the four regions are $0.12,0.13,0.13,0.14$, respectively. The published values of $E(B-V)$ are 0.15 (Oey), 0.01 (Hill et al.), 0.16 or 0.11 (Hill et al. and Oey, respectively for $\mathrm{LH} \mathrm{83)}$, and 0.08 (Oey) for the four regions in the same order as above. The published values are typically based on only tens of observed stars and have large uncertainties (Hill et al. quote uncertainties of $\sim 0.08$ ). The values we 
attribute to Oey are medians taken from her list of reddenings to individual stars in the regions. We find no systematic differences between our values and the published ones, although the difference in one case (LH 54) is slightly larger than the uncertainty (but only by a factor of 1.5). This difference may be unimportant because Hill et al. note that their data for this region contained significant scatter among reddenings and many negative reddening values, suggesting that there may have been a problem with the data. Other than the LS 54 field, the others are entirely in agreement with the published data, although the large uncertainties preclude more detailed comparisons.

\subsection{Comparison to the OGS's Foreground Extinction Measurements}

We proceed to test whether the extinctions derived for low $T_{E}$ stars are consistent with previous studies. The only comparable study is that by Oestreicher, Gochermann, \& Schmidt-Kaler 1995 (hereafter, OGS) which used Galactic stars in the direction of the LMC to measure the foreground extinction toward the LMC. We divide our low $T_{E}$ sample into foreground Galactic stars and LMC stars by attributing the large vertical plume of stars seen in the Hess diagram at $B-V \sim 0.6$ and $V<18$ to Galactic stars (cf. Figure 1 from HZT, and references therein). By examining the extinction values of the foreground and LMC populations separately and comparing them to the measured foreground (Galactic) extinction from OGS, we can determine whether (1) our foreground values are in agreement with OGS, (2) whether our LMC values are consistent with $($ i.e., $\geq$ ) the foreground extinction values, and (3) whether the metallicity difference between the Galactic and LMC populations grossly affects the derived extinctions.

We begin by comparing our extinction values for the Galactic and LMC low $T_{E}$ populations with the OGS values. Unfortunately, the comparison is not ideal because the OGS data do not sample the region as densely as do our data. Figure 8 shows the comparison between reddenings to individual OGS foreground stars and values inferred from an extinction map constructed using our foreground stars. There is no correlation visible (although the range of values along the axes are similar). The scatter demonstrates the large uncertainties in the values along any single line-of-sight.

Although the scatter in individual lines-of-sight is large, over large areas the extinction map may be reproducible and reliable. To test this possibility, we first compare the distribution of OGS extinction values, including all OGS stars across the entire LMC to improve the statistics (no detectable difference was seen when only using the foreground stars within our survey region), to the distribution of extinction values derived for the foreground stars in our sample in Figure 9. Although the $A_{V}$ values from the OGS data are somewhat more widely distributed than the values we infer from our foreground population, the modes of the two populations are similar (both $\sim$ $0.11 \mathrm{mag})$. The wider OGS distribution may imply either that the uncertainties are larger in the OGS data than in our data, that we have underestimated $A_{V}$ for some of the stars in our survey, or that the region sampled by OGS included regions of higher foreground extinctions. The quoted uncertainties in the OGS data are mostly $<0.06$ mag, insufficient to account for the larger spread in values. This conclusion is further supported by the rough similarity in the fraction of points with $A_{V} \leq 0$ in both datasets. In bulk, the statistics of the foreground extinction as derived from our data and OGS's are quite similar.

We compare the $A_{V}$ distributions for the Galactic and LMC stars in the inset of Figure 9. There is little difference in the distributions except for the more significant tail of higher extinction values in the LMC stars. This agreement demonstrates that there are no gross differences between the extinctions derived from the different set of stars due to physical differences, like metallicity, and that the LMC data are consistent with the extinction inferred from the foreground data (from both the OGS data and our own).

Although statistically the extinctions inferred from the OGS data and our own are similar, we need to confirm whether we can accurately trace the spatial distribution of dust to validate out extinction map. To improve the statistics for the comparison between the OGS observations and ours, we compare the extinction map produced from the OGS data to that obtained using all of our low $T_{E}$ stars (the LMC stars should trace the foreground extinction pattern in addition to any pattern imposed by dust internal to the LMC). 
Pixel values in our low $T_{E}$ extinction map are tagged according to the extinction value given by the OGS map for the corresponding location. We then bin the data using the OGS extinction values and set the bin size to include extinction values from at least 200 different pixels within each bin. The average of our extinction values within each bin is plotted versus the OGS extinction value in Figure 10. There is a clear correlation between our low $T_{E}$ extinction measurement and the OGS data, demonstrating that the bulk properties of the OGS map and our extinction map are reproduced and that our analysis is recovering the spatial behavior of foreground extinction in this region. There are a few discrepant values in bins at low values of the OGS extinction, but the pixels responsible are localized in our map. Because of this spatial localization, the discrepancy is either due to slightly erroneous photometry in a limited region (e.g., poor aperture correction) or to a patch of extinction internal to the LMC extinction that is not represented in the OGS foreground extinction map. We conclude that the strong correlation demonstrates that we recover the spatial distribution of extinction and that there is no dominant component of extinction internal to the LMC that affects the low $T_{E}$ stars.

Despite the strong correlation, the relation between the OGS results and ours is similar to that seen in Figure 9 (our extinction values < OGS extinction values). This offset may be the result of a slight bias in our method when applied to the cool stars (e.g., inclusion of some intrinsically higher $T_{E}$ stars for which $A_{V}$ is underestimated or the exclusion of some higher $A_{V}$ stars due to our photometric selection), or it may be indicative of unresolved structure in the OGS map. If areas of high extinction are small on the sky, then a map produced from sparsely sampled data would occasionally include large inferred areas of high extinction (large areas of low extinction would also be present but would be a better representation of "reality"). When such a map is compared to a map with higher resolution, a bias in the observed sense would exist. We cannot determine which of these two scenarios is responsible for the systematic discrepancy we observe, but we conclude that to within this possible slight bias our extinction determinations for low $T_{E}$ stars recover the statistical properties and spatial behavior of the foreground extinction found in previous determinations of the foreground extinction.

\subsection{Comparison to the IRAS $100 \mu \mathrm{m}$ Im- age}

To determine whether the medianing we perform to mitigate the uncertainties and create the extinction map results in a physically meaningful map, we compare our map to images of the far infrared emission. The far IR emission comes from the dust, but the emitted flux depends on the dust temperature and hence on the local radiation field. Therefore, although $100 \mu \mathrm{m}$ flux is expected to be a tracer of dust, we do not expect it to be a complete map of the extinction. We have extracted the IRAS $100 \mu \mathrm{m}$ image of the area and present it, in conjunction with the extinction map from the high $T_{E}$ stars and the density-weighted image of main sequence stars with $V<16.5$ and $B-V<0.5$, in Figure 11 (B3 and earlier main sequence stars). We apply our mask to the IRAS image to produce an image that exactly matches the area for which we have optical data. Most of the prominent dust features are visible in both the extinction map and the IRAS data, which further confirms that structures seen in the extinction map match true dust structures.

There are only a few instances where significant $100 \mu \mathrm{m}$ flux exists without strong extinction or where strong extinction exists without significant $100 \mu \mathrm{m}$ flux. Most of the differences can be traced to the presence or lack of nearby young stars. For example, the extinction map shows a long vertical filament in the upper portion of the left half of the survey region. In extinction, this filament does not have strong variations along the filament. However, the $100 \mu \mathrm{m}$ emission is strongly peaked near the lower portion of the filament, coincident with the presence of young stars that provide the UV flux needed to heat the dust. A more extreme example is the small, brightest knot of $100 \mu \mathrm{m}$ emission in the upper half of the left side of the region, which has no noticeable extinction consequence but is coincident with a group of young stars. Highly localized dust may be diluted in our map by the medianing we do, but because this area includes a large number of OB stars we have not diluted the extinction at this specific location. These examples demonstrate that both the column density of dust and the presence of 
heating sources are important in determining the $100 \mu \mathrm{m}$ morphology, but that in general the extinction features determined from medianing over several stars per pixel are confirmed by the $100 \mu \mathrm{m}$ emission.

Although strong IR emission appears to require the presence of coincident young stars, young stars are not always indicative of a strong $100 \mu \mathrm{m}$ emission feature. The dominant concentration of young stars (of both the $\mathrm{O}$ and B stars) within the survey region is in the middle of the left side of the region. However, this area is a one of the lowest areas of $100 \mu \mathrm{m}$ flux, presumably because there is less dust there than in other areas - as suggested by the $A_{V}$ map. Perhaps the young stars have removed the dust along the line of sight by blowing a bubble out of the disk plane in our direction (cf. MacLow and McCray 1988). Such a scenario would also be in agreement with the ring-like dust morphology surrounding this region seen both in the extinction map and the $100 \mu \mathrm{m}$ image. Observations of the ionized gas in this region are also qualitatively consistent with this scenario (Hunter 1994). If the bubble is as large in the line-of-sight direction as in the cross direction $(\sim 1 \mathrm{kpc})$, then it has blown out of the LMC disk plane.

We quantify the connection between dust, $100 \mu \mathrm{m}$ flux, and young stars by examining the correlation among pixel values in the various images. By identifying pixels in the lower panel of Figure 11 that contain 2 or more OB stars (about $16 \%$ of all pixels), we find that $71 \%$ of all OB stars are contained in these pixels, but that only $21 \%$ of the $100 \mu \mathrm{m}$ flux is contained within these same pixels. Each pixel corresponds to a projected size of $20 \mathrm{pc}$ (the dust map is smoothed using a Gaussian of 3 pixels full-width at half maximum). Although there is a slight increase in $100 \mu \mathrm{m}$ flux over that expected from a uniform distribution (21\% vs. 16\%), most of the IR flux is not coming from the regions of highest OB star concentrations. Pixels without a single OB star contain $54 \%$ of the $100 \mu \mathrm{m}$ flux. If instead we divide the region on the basis of pixels that have higher than median $100 \mu \mathrm{m}$ emission $(12.1 \mathrm{MJy} / \mathrm{sr} ; 50 \%$ of the pixels), we find that this area contains $75 \%$ of the OB stars. These numbers quantify the visual impression from Figure 11 that regions with the highest $100 \mu \mathrm{m}$ flux lie near sites of OB stars, but that the local presence of OB stars does not im- ply strong $100 \mu \mathrm{m}$ emission. These arguments are complicated by the unknown three-dimensional nature of the problemk and the fact that OB stars can heat dust hundreds of pc away.

The relative distribution of young stars and $100 \mu \mathrm{m}$ flux within this region illustrates why it has been difficult to resolve the issue of the heating source of dust in other galaxies. Although the $100 \mu \mathrm{m}$ flux correlates in general with young stars (i.e., more $100 \mu \mathrm{m}$ flux implies more young stars), a substantial fraction of the $100 \mu \mathrm{m}$ flux is diffuse (i.e., coming from beyond the sites of OB stars) and there are regions rich in OB stars with little or no $100 \mu \mathrm{m}$ flux. It is evident from the distribution of the two components that $100 \mu \mathrm{m}$ flux cannot be used to trace star formation in detail (even if globally correlated), and that there may be a large dispersion in global characteristics as well, although we cannot address that issue with these data. Our data directly demonstrate the scatter in local properties, but global properties may still be correlated if the galactic radiation field is dominated by the radiation from OB stars. The local scatter in any relation between young stars and $100 \mu \mathrm{m}$ flux is evident if one considers the central region of LMC 4 (which is currently nearly empty of dust and hence of $100 \mu \mathrm{m}$ emission). The main arc of young stars in this region has 17 pixels that contain as many OB stars as the densest pixel corresponding to the bright $100 \mu \mathrm{m}$ emission knot just above the arc. If there was dust within the arc of young stars one would expect the flux to be at least 17 times greater than that coming from the knot, which is evidently not the case. Therefore, although large $100 \mu \mathrm{m}$ fluxes are likely to be an accurate indication of star formation, the converse is less likely to be true and $100 \mu \mathrm{m}$ flux has a large dispersion as a local tracer of recent star formation within galaxies. A complete treatment (UV photometry to measure ionizing flux, optical, HI, and a variety of infrared bands) is necessary to make further progress in resolving the full energy budget of this process, but is beyond the scope of the current study.

\subsection{Variation with Stellar Population}

We have demonstrated that the extinction measurements for the low and high $T_{E}$ stars are in agreement with previous measurements of extinction and emission from dust. Therefore, we can 
begin to compare with some confidence the relative distributions of dust that affect the low and high $T_{E}$ stars. The upper and lower panels of Figure 5 demonstrate that the line-of-sight extinctions are generally much lower for the low $T_{E}$ stars. This impression is confirmed by the $A_{V}$ histograms for the two stellar populations (Figure 12). The trend of declining mean extinction with declining $T_{E}$ (and presumably with increasing mean age) is also present within subsets of the high $T_{E}$ data. In Figure 13 we plot the $A_{V}$ distribution for four subsets of high $T_{E}$ stars. There are several trends evident as $T_{E}$ increases: (1) the number of very low $A_{V}(<0.1 \mathrm{mag})$ stars decreases, (2) the peak of the distribution narrows and stays at an almost constant $A_{V}$, and (3) the tail of high $A_{V}$ values becomes increasingly more significant as $T_{E}$ increases. Even within the set of high $T_{E}$ stars, higher values of extinction are more common for the hotter, younger stars. To test this result, we also plot the HZT results using the classical reddening-free method (the $\mathrm{Q}$ method). Because there are fewer stars in the HZT sample, we divide it into only two subsamples at $V=15$. As luminosity (and $T_{E}$ ) increase, this sample reproduces both the smaller relative number of low $A_{V}$ values and the larger relative number of high $A_{V}$ values.

Even though the non-Gaussian $A_{V}$ tail affects a small fraction of the total stellar population, it can have serious effects on certain observations and suggests that the dust is highly localized. Clumpy regions of high extinction effectively flatten the extinction curve (in the extreme case where one has only completely optically thick clumps, some stars will be extincted out of the sample, but the colors of the observed stars may not change; cf. Szomoru \& Guhathakurta 1999). This flattening of the effective extinction curve for the LMC as a whole is countered by our observations that the bluer stars are more heavily extincted than the red stars, which steepens the effective extinction curve. The degree to which these two effects cancel depends on the detailed distribution of dust and stars, which are still poorly determined in detail. Lastly, because of the non-Gaussian $A_{V}$ tail, general properties of the $A_{V}$ distribution, such as the mode, may not truly reflect the amount of light lost from the entire population due to the dust.

To demonstrate the error introduced by using the mode of the $A_{V}$ distribution to correct photometry, we compare the amount of light extincted in the $12000 \mathrm{~K}<T_{E}<14000 \mathrm{~K}$ sample to that extincted in the $22000 \mathrm{~K}<T_{E}<45000 \mathrm{~K}$ sample. One might at first believe that fractionally the extincted light would be comparable because the modes of the two distributions are quite similar (cf. Figure 13), particularly if the available data were insufficient to significantly populate the high $A_{V}$ tail in the hotter sample. However, using the full distribution of $A_{V}$ 's and assuming that all of the stars within the temperature range have the same luminosity (i.e., no $A_{V}-T_{E}$ correlation within the bins), we find that $29 \%$ of the V-band light is extincted from the cooler sample vs. $42 \%$ from the hotter sample. As stated before, this progression makes the effective extinction curve for the galaxy as a whole (ignoring scattering in unresolved galaxies) steeper (i.e., smaller $R_{V}$ ). We conclude that even among high $T_{E}$ stars it is critical to measure the distribution of $A_{V}$ to accurately correct for extinction.

Finally, we compare the extinctions from the hot and cool stellar populations by comparing the extinction maps pixel by pixel (Figure 14). Again, we bin and average (so that each bin contains values from at least 200 pixels). The positive correlation in Figure 14 demonstrates that the dust affecting the hot population also affects the cool population, but the correlation is not one-toone. To understand this correlation, we examine a model of the relative distributions of stars and dust in which the LMC dust is in a plane of scaleheight $\ll$ scaleheight of the cool stars. In such a geometry, half of the cool stars are only extincted by Galactic foreground dust. The LMC dust partially fills the central LMC disk plane, so a yetunspecified fraction of the stars behind the LMC midplane are extincted by the Galactic foreground plus the LMC disk and the remainder are seen through holes in the LMC disk and therefore only extincted by the Galactic foreground dust. The extinction due to the LMC disk is taken to be the extinction predicted from the high $T_{E}$ stars minus the mean foreground extinction and constrained to be $\geq 0$. The foreground extinction is taken to be $0.173 \mathrm{mag}$ (the average from the extinction map constructed from the OGS data for the relevant region of the LMC), and we plot in Figure 14 the results of adopting dust "filling factors" for the 
LMC dust of $0.0,0.5$, or 1.0. A filling factor of 1 corresponds to the case where the disk extinction is exactly that given by the high $T_{E}$ extinction map, while a filling factor of 0.5 corresponds to the case where half of the lines-of-sight are clear and half are extincted as described by the high $T_{E}$ extinction map. This simple model with a filling factor of 1.0 successfully reproduces the behavior in the Figure. The extinction predicted by the model toward the left of the Figure is lower than observed, but only by 0.02 mag. If one adds 0.02 mag to the foreground model (to resolve this disagreement), then the models with a filling factor $\sim 0.5$ work equally well. In either case, we conclude that a model where the low $T_{E}$ stars have a significantly larger scaleheight than both the OB stars and the dust and where the dust structure recovered from the high $T_{E}$ stars is taken to represent that of the midplane sheet works well in reproducing both the low and high $T_{E}$ extinction behavior.

\subsubsection{Some Implications for the Distance Scale}

One area where accurate extinction corrections are critical is distance scale work. The populationdependent extinction observed in the LMC is also presumably present in other galaxies and can influence observations of those galaxies. As such, it becomes important not only to measure the extinction local to the source of interest (e.g., Cepheid or supernovae), but also to measure the distribution of extinctions for a correspondingly similar stellar population. Both of these goals obviously become increasingly difficult the more distant a galaxy is, but they can even be important for studies of the LMC. Inappropriate reddenings adopted using mismatched stellar populations can affect the derived distance to the LMC, which is often a key calibrating galaxy. Reddening estimates are generally determined from OB stars (cf. HZT) which are bright and for which unambiguous reddening measurements can be obtained using the straightforward application of the classical reddening free color technique (the Q method). However, the results in this paper illuminate several key difficulties with this approach. First, the reddenings determined in this way should not be applied to Pop II standard candles (cf. red clump stars and red giant tip stars). Second, even among the high $T_{E}$ stars, there can be differences in the distribu- tion of extinction values. Third, because the distribution can be highly non-Gaussian, significant errors can be introduced by using certain statistical properties (e.g., mode) rather than the entire $A_{V}$ distribution to correct the photometry.

We do not intend here to provide a complete review of how variable extinction will affect each distance method. However, a few examples will demonstrate that the magnitude of the effect is comparable to the degree of disagreement between various published results. First we consider the use of the LMC to zero-point the Cepheid P-L relation. We have already demonstrated in Figure 13 that even among stars with $T_{E}>12000 \mathrm{~K}$ (the young stellar population and therefore appropriate for Cepheids) there are differences in the distribution of extinction values. Any determination of the extinction will be dominated by the coolest stars included because there will be many more of them. For example, the mean visual extinction obtained using all of the stars with $T_{E}>12000$ $\mathrm{K}$ is 0.42 and the mean using only those stars with $T_{E}>22200 \mathrm{~K}$ is 0.60 . Even in the I band, where the effects of extinction are more moderate, this difference corresponds to 0.11 mag. Assuming that we have chosen the appropriate subpopulation to match to the Cepheids, the nonGaussian tail creates significant differences between the mean and median $A_{V}$. For example, for the $T_{E}>22000 \mathrm{~K}$ sample the mean and median $A_{V}$ are 0.57 and 0.44 , respectively. Which should one apply? Because of the unknown small scale structure of the dust, a photometric correction will be statistical and the properties of the $A_{V}$ distribution are critical. As discussed in detail by Madore and Freedman (1998), the issue of extinction in relation to the distance scale has been extensively investigated and the most promising techniques are those that sidestep the issue by either using reddening-free quantities or by moving as far into the infrared as possible to minimize the effect of extinction. Nevertheless, investigators are often left with no option but to estimate the extinction from "local" stars. We have shown here that this approach has possible systematic problems.

Our results suggest another way to mitigate the influence of dust. Because the extinction is lower for the colder, older stars, using Pop II standard candles is analogous to going to the in- 
frared for Pop I standard candles. In a previous study (Stanek, Zaritsky, \& Harris 1998), we used our survey data to investigate the small distance modulus, 18.08, obtained by a previous study of field red clump stars (Udalski et al. 1998). Using the map of HZT we were able to select low extinction regions and then correct the photometry for the extinction that was present $\left(A_{I}=0.31\right.$, $E(B-V)=0.17)$. That analysis confirmed the small distance modulus determined by Udalski et al. . Since that study, the discussion in the literature has focussed on the magnitude of the age and metallicity corrections to the red clump magnitude. Corrections derived from stellar models (Cole 1998; Girardi et al. 1998) revised the red clump distance modulus to 18.3 , which is still below the "standard" value of 18.5 but within the uncertainties. The difficulty with theoretical corrections is that they are based on models of a complex phase of stellar evolution. Empirical studies of the red clump conclude that the age and metallicities are more moderate (Udalski 1998a, 1998b) and that the distance modulus is still $\sim 18.1$. The difficulty with empirical corrections is that they depend on the complicated interplay of systematic uncertainties in the various distance estimates used to calibrate the age and metallicity dependence of the red clump luminosity. Our study does not address the age and metallicity corrections, but rather returns to the issue of the extinction correction.

The HZT extinction map Stanek et al. used is based on observations of OB stars and here we find that such a map is not an accurate representation of the reddening to red clump stars. Our low $T_{E}$ extinction map is ideal in correcting for the extinction because red clump stars are within the $T_{E}$ range included in the construction of this map. Our new data suggest that $A_{V}$ for the field red clump stars in regions of low extinction (as selected by Stanek et al. ) is $\sim 0.1$ and so $A_{I} \sim 0.06$, which increases the derived distance modulus by 0.25 mag to 18.32 for the data presented by Stanek et al. . If age and metallicity corrections of the magnitude suggested by Cole (1998) or Girardi et al. (1998) are then applied ( $0.2 \mathrm{mag})$, the red clump distance modulus can be made as large as 18.5, in excellent agreement with the "standard" value. Whether the latter corrections are necessary is not addressed by our data and should not be inferred from the agreement with the standard distance value.

We do not want to leave the reader with the impression that the correction for populationdependent extinction removes all of the discrepancies among LMC distance determinations. There remain several arguments for an LMC distance modulus that is smaller or larger than the standard. Some analyses of RR Lyrae observations suggest a small LMC distance modulus (Walker 1992a; Layden et al. 1996), and an erroneous reddening correction is not responsible for artificially decreasing the apparent distance because the reddenings for cluster RR Lyrae are derived from color-magnitude diagrams. The extinctions to these clusters are an accurate measure of the extinction toward the RR Lyrae (cf. Walker 1992b for an example). Only two of the seven clusters used by Walker 1992a have $E(B-V)>0.1$, in agreement with our generally low extinctions to older populations. However not all RR Lyrae measurements distances are small, RR Lyrae distances using Hipparcos-based calibrations have given much larger distance moduli (18.65, Reid 1997; 18.63 Gratton et al. 1997). Some other methods, for example the analysis the SN 1987A ring, which again is not affected by the problems discussed here, can result in a smaller distance modulus (cf. $\lesssim 18.37$ (Gould and Uza 1998)), but can also result in larger values (cf. 18.5, Panagia et al. 1991; 18.43, Sonneborn et al. 1997). Specifically for the red clump stars, Udalski (1998b) presents data from LMC clusters, for which the extinctions are small, and obtains a low value of the distance modulus that is in agreement with field red clump distance modulus with the original extinction correction. The disagreement between the field and cluster red clump distances that results from our new extinction correction is not easily resolved. The clusters have low extinctions, so even if the adopted extinction is incorrect, the effect on artificially depressing the distance modulus is minor. Presumably the cluster stars have similar ages and metallicities to the field stars, so the resolution will not come from differential age and metallicity corrections.

These unexplained discrepancies still render the distance measurement to the LMC uncertain. However, we conclude that population-dependent extinction is yet another issue that must be ad- 
dressed as one measures distances and that extinction differences between populations can result in errors comparable to other, better appreciated, sources of systematic error.

\subsubsection{Some Implications for Studies of Stellar Populations}

The results presented here also have implications for the observation of stellar populations in both resolved and unresolved galaxies. The study of resolved populations has been advanced in the last few years by deep HST color-magnitude diagrams with many thousands of stars, and ongoing ground-based surveys that will provide colors and magnitudes of millions of stars. Aside from identifying rare stellar populations that can provide key clues to the star formation history of the galaxy, the large samples allow precise determinations of the apparent positions and dispersions of populations, like the red giant branch, which in turn can constrain the range of ages and metallicities present in the system. The dominant source of error in such analyses will no longer be statistical but systematic. The extinction correction is a principal potential source of such systematic uncertainties. We were in fact driven to this analysis by our inability to fit the main sequence and red giant branch simultaneously when applying a single distribution of extinctions to a portion of our LMC data. Any detailed, precision analysis of stellar systems that includes relatively recent star formation must account for population-dependent extinction.

The situation is even more complex in unresolved galaxies. As previous studies have stressed (cf. Witt \& Gordon 1996), the interpretation of the measured surface brightnesses is complicated by light scattered back into the line-of-sight and multiple scatterings. Neither of these issues are addressed by the current data on the resolved LMC because any scattered light is removed as local background to each star. Ignoring scattering, we can estimate the amount of light lost due to extinction from both the hot and cool stellar populations in the LMC to determine how an unresolved galaxy with such stellar populations might be perceived. In the $V$ band, extinction has removed $28 \%$ of the light from the hot stars but only $13 \%$ of the light from the cool stars. In the $B$ band, extinction removes $33 \%$ of the light from the hot stars but only $16 \%$ of the light from the cool stars. Correcting the apparent luminosity of the galaxy using the extinction appropriate for $\mathrm{OB}$ stars (or alternatively from emission line extinction measurements $(\mathrm{H} \alpha / \mathrm{H} \beta)$, which are likely to be similar to the $\mathrm{OB}$ values), will overestimate the galaxy luminosity by $\sim 15 \%$ and introduce a color correction that is about 2 times larger than that appropriate for the bulk of the stellar population. Although investigators have been aware of such issues, the difficulty in dealing with the large unknown number of parameters has led to very simple treatments. Our discussion here provides an indication of the degree of uncertainty that such simplifying assumptions may impose.

\section{Conclusions}

We have fit colors derived from stellar models to 4-filter photometry of LMC stars to derive effective temperatures and visual extinctions. We find that there are two populations of stars for which we can reliably recover the visual extinction, stars with $5500 \mathrm{~K}<T_{E}<6500 \mathrm{~K}$ and stars with $T_{E}>12000 \mathrm{~K}$. Various tests demonstrate that we are not strongly sensitive to metallicity and that we recover extinctions in agreement with previous studies to within a dispersion of $\sim 0.1$ to $0.2 \mathrm{mag}$ for individual stars. More precise extinction values are derived by combining the results from many stars and producing extinction maps of the region. Our principal result is that there is a significant difference in the extinction values derived for the two samples of stars, with the hotter stars being more highly extincted on average.

The difference in extinction properties as a function of stellar population is not entirely unexpected, but we present direct evidence and quantify the effect for the LMC. We test for biases by comparing to previously published extinction measurements and to $100 \mu \mathrm{m}$ images of the region. We find that in general the $100 \mu \mathrm{m}$ emission features trace the extinction morphology, but that there are a few exceptions. These few exceptions can be traced to the presence or absence of young stars that heat the dust. We identify several locations where young stars have heated the dust so that it emits strongly at $100 \mu \mathrm{m}$ and locations where there are young stars, but apparently no dust because there is no $100 \mu \mathrm{m}$ emission. We conclude 
that significant $100 \mu \mathrm{m}$ flux correlates with young stars, but that young stars do not necessarily imply significant $100 \mu \mathrm{m}$ flux and that $100 \mu \mathrm{m}$ flux should only be used as a star formation tracer with caution. This is in agreement with the results of Walterbos and Schwering (1987) for M 31.

We presented a simple model for the distribution of stars and dust along the line of sight. Previous results (HZT) demonstrate that the scaleheight of the dust is about twice that of the OB stars. Because the low $T_{E}$ stars have different extinction properties, we hypothesize that the scaleheight of this populations is $\gg$ than that of the dust. Such a model implies that half of the low $T_{E}$ stars are completely in front of the LMC dusty disk and that half are extincted by the extinction at that location in the disk (given by the high $T_{E}$ extinction map). We then compare the expectation from this model with the spatial correlation between low and high $T_{E}$ extinction measurements. We find that this model fits the data remarkably well and provides a simple explanation for the different extinction properties of the two samples.

We explore some ramifications of the populationdependent extinction. In particular, we discuss its effect on distance measurements of the LMC. The expected behavior is in the correct sense to help resolve the discrepancy between distance moduli found using various stellar populations (cf. RR Lyrae, red clump stars, and Cepheids). We present how the distance measured using field red clump stars may have been biased downward by using reddening measurements obtained from $\mathrm{OB}$ stars, rather than from red clump stars, in the same region. Taking the mean extinction obtained from the cooler stars, which are directly comparable to the red clump stars in temperature, the distance modulus inferred is 0.2 mag larger than previously derived, in better agreement with values obtained from observations of Cepheids. However, the Cepheid distance measurements may also be affected by population-dependent extinction variations because young stars are subject to greater and more variable extinction, making determination of the $A_{V}$ distribution, rather than a mean or median, key in properly accounting for the effects of extinction. We discuss how observations of galaxies in general may be affected by the population-dependent reddening. Treating this section of the LMC as a complete independent galaxy, we derive that nearly twice as much of the light from the hotter stars is removed by extinction than of the cooler stars. This effect results in an effective extinction curve steeper than the true curve.

We conclude by stressing that dust affects different stellar populations differently. Simple models of obscuring planes or well-mixed stars and dust do not accurately represent the physical situation. Whether this gravely affects our interpretation of observations is still not fully understood. We illustrate that in certain cases ignoring the results presented here could have serious consequences. We continue to reduce the data obtained for the LMC and SMC as part of our survey and soon should be able to examine the distribution of dust on even larger scales. Then we can address whether the lack of dust in the center of the starforming region in LMC-4 is common for bubbles and superbubbles, how the dust is distributed though the disk of the LMC, how it is distributed in the bar, and whether similar distributions are observed in the SMC. These data provide a unique opportunity to explore the relative distributions of stars and dust within galaxies and are a necessary first step in properly interpreting the highly populated color magnitude diagrams that are becoming available for the Clouds.

We thank Rob Kennicutt and Ann Zabludoff for detailed comments and suggestions. DZ acknowledges financial support from an NSF grant (AST9619576), a NASA LTSA grant (NAG-5-3501), a David and Lucile Packard Foundation Fellowship, and an Alfred P. Sloan Fellowship.

\section{REFERENCES}

Astronomical Almanac, 1981, (US Government Printing Office: Washington, D.C.)

Cardelli, J.A., Clayton, G.C., and Mathis, J.S. 1989, ApJ, 345, 245

Carrasco, L., Strom, S.E., and Strom, K.M. 1973, ApJ, 182, 95

Cole, A.A. 1998, ApJ, 500, L137 
Devereux, N.A., and Young, J.S. 1990, ApJ, 350, L25

Devereux, N.A., Duric, N., and Scowen, P.A. 1997, AJ, 113, 236

Feast, M.W. and Catchpole, R.M. 1997, MNRAS, 286, L1

Girardi, L., Groenewegen, M.A.T., Weiss, A., and Salaris, M. 1998, MNRAS, 301, 149

Gratton, R.G., Pecci, F.F., Carretta, E., Clementini, G., Corsi, C.E., and Lattanzi, M. 1997, ApJ, 491, 749

Harris, J., Zaritsky, D., Thompson, I. 1997, AJ, 114,1933

Harris, J., and Zaritsky, D. 1999, AJ, in press

Hill, R.J., Madore, B.F., and Freedman, W.L. 1994, ApJ, 429, 192

Houk, B., and Smith-Moore, M. 1988, Catalogue of Two-Dimensional Spectral Types for the HD Stars. Vol. 4 (Univ. of Michigan, Ann Arbor)

Huizinga, J.E. 1994, Ph.D. thesis, Univ. Groningen

Hunter, D.A. 1994, AJ, 107, 565

Johnson, H.L., and Morgan, W.W. 1953, ApJ, 117,313

Jones, H., Davies, J.I., and Trewhella, M. 1996, MNRAS, 283, 316

Koornneef, J. 1982, A\&A, 107, 247

Kuchinski, L.E., Terndrup, D.M., Gordon, K.G., and Witt, A.N. 1998, AJ, 115, 1438

Lanz, T. 1986, A\&AS, 65, 195

Layden, A.C., Hanson, R.B., Hawley, S.L., Klemola, A.R., and Hanley, C.J. 1996, AJ, 112, 2110

Lejeunne, Th., Cuisinier, F., and Buser, R. 1997, A\&AS, 125, 229

Lonsdale-Persson, C.J., and Helou, G. 1987, ApJ, 314,513

Lucke, P.B., and Hodge, P.W. 1970, AJ, 75, 171
MacLow, M.-M., McCray, R. 1988, ApJ, 324, 776

Madore, B.F., and Freedman, W.L. 1998, ApJ, 492, 110

Mermilliod, J.C. 1986, A\&AS, 24, 159

Nandy, K., Morgan, D.H., Willis, A.J., Wilson, R., Gondhalekar, P.M., and Houziaux, L 1980, Nature, 283, 725

Nandy, K., Morgan, D.H., and Houziaux, L. 1984, MNRAS, 211, 895

Oey, M.S. 1996, ApJ, 465, 231

Pagel, B.E.J., Edmunds, M.G., Fosbury, R.A.E., and Webster, B.L. 1978, MNRAS, 184, 569

Panagia, N., Gilmozzi, R., Macchetto, F., Adorf, H.-M., and Kirshner, R.P. 1991, ApJ, 380, L23

Reid, I.N. 1997, AJ, 114, 161

Sauvage, M., and Thuan, T.X. 1992, ApJ, 396, 69

Schild, R.E. 1977, AJ, 82, 337

Sonneborn, G., Fransson, C., Lundqvist, P., Cassatella, A., Gilmozzi, R., Kirshner, R.P., Panagia, N., and Wamsteker, W. 1997, ApJ, 477, 848

Stanek, K.Z., Zaritsky, D., and Harris, J. 1998, ApJ, 500, L141

Straižys, V. 1992, Stellar Photometry (Pachart, Tucson)

Szomoru, A. and Guhathakurta, P. 1999, in press (astro-ph/9901422)

Tully, R.B., and Fouqué, P. 1985, ApJS, 58, 67

Udalski, A., Szumanński, M., Kubiak, M., Peitrzyński, G., Woźniak, P., and Zebruń, K. 1998, Acta Astron., 48, 1

Udalski, A. 1998a, Acta Astron., 48, 113

Udalski, A. 1998b, astro-ph/9807095

Walker, A.R. 1992a, ApJ, 390, L81

Walker, A.R. 1992b, AJ, 104, 1395

Walterbos, R.A.M., and Schwering, P.B.W. 1987, A\&A, 180, 27 
Witt, A.N., and Gordon, K.D. 1996, ApJ, 463, 681

Young, J.S., Schloerb, F.P., Kenney, J.D., and Lord, S.D. 1986, ApJ, 304, 443

Zaritsky, D., Harris, J., and Thompson, I. 1997, AJ, 114, 1002

Zaritsky, D., Shectman, S.A., and Bredtahuer, G. 1996, PASP, 108, 104

This 2-column preprint was prepared with the AAS LATEX macros v5.0. 


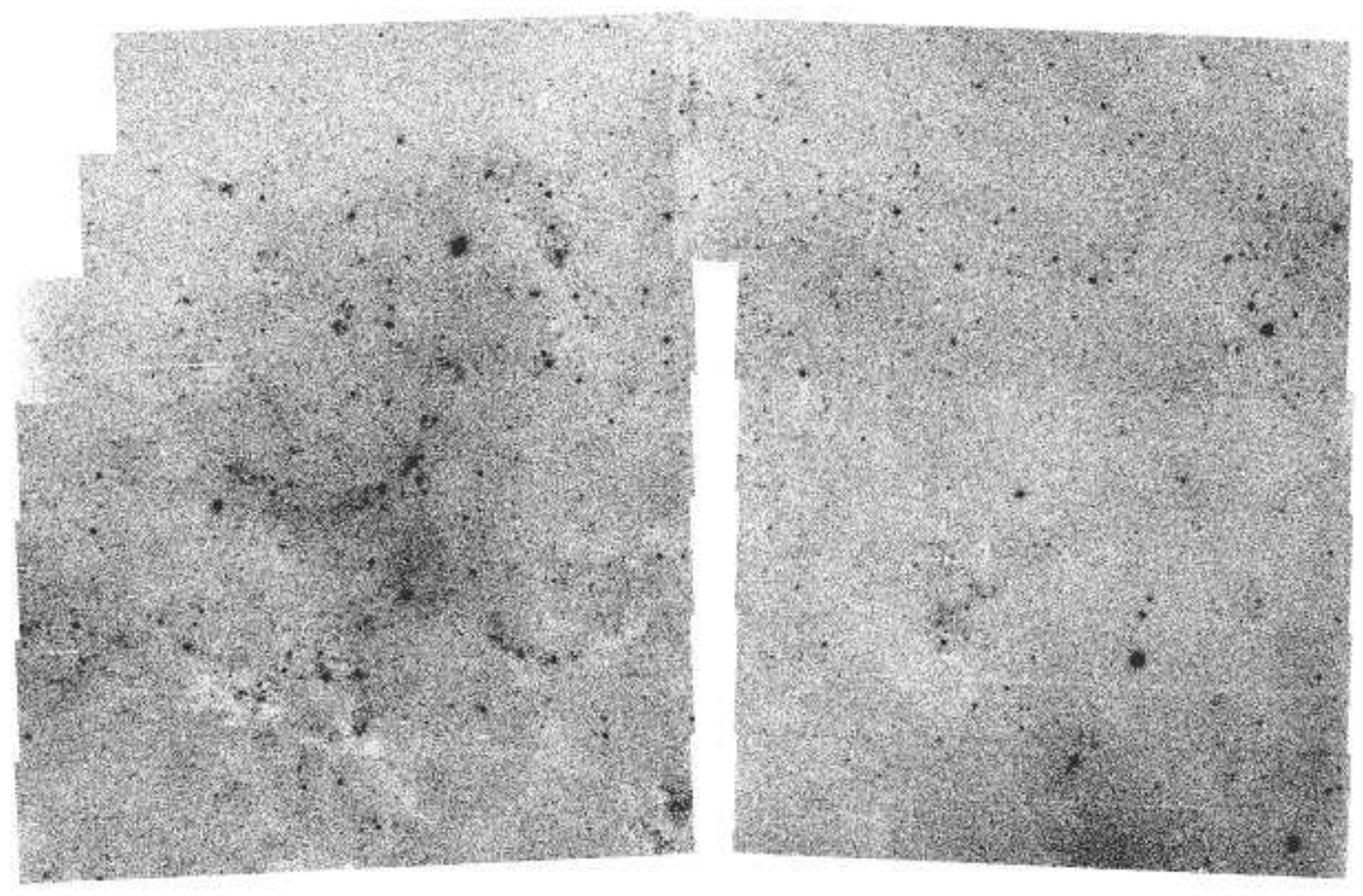

Fig. 1.- The stellar density plot (for stars with $V<21$ ) for the region in the LMC with reduced photometry from the Magellanic Cloud Photometric Survey. The central coordinates are roughly $\alpha=5^{h} 20^{m}$ and $\delta=-66^{\circ} 48^{\prime}$, the image is $\sim 4^{\circ}$ wide by $2.7^{\circ}$ tall, with North at the top and East to the left. Each "pixel" in this plot corresponds to $21^{\prime \prime}$. 


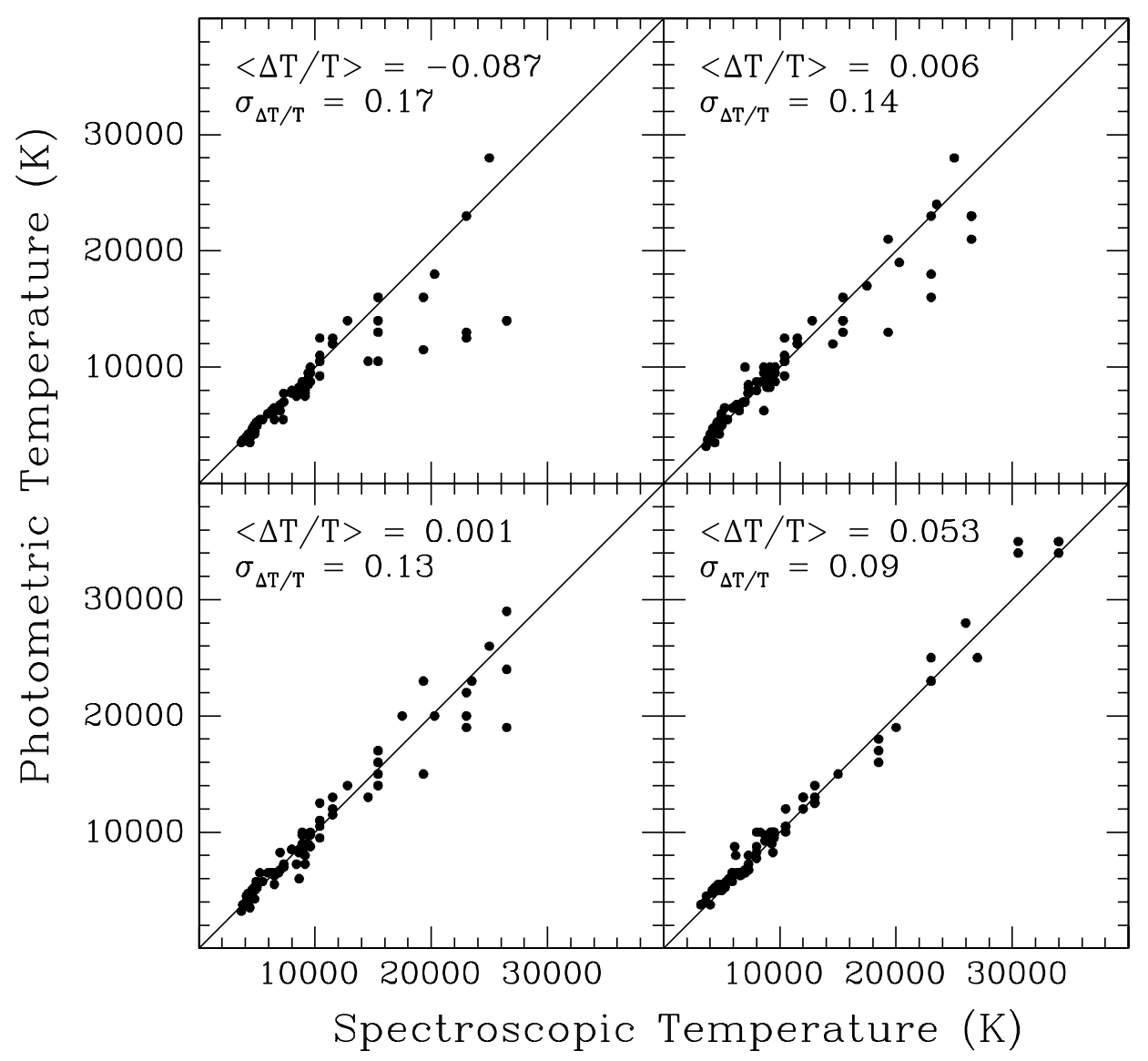

Fig. 2.- Comparison of photometrically derived effective temperature with spectroscopically derived effective temperature. Each panel depicts the results for standard stars from the application of an algorithm that matches stellar models to data. The results in the upper left panel were obtained with neither a correction for extinction or a photometric zero point shift between the standard stars and the models. The results in the upper right panel were obtained from models that include extinction as a fitting parameter, but do not include a zero point photometric shift. The results in the lower left panel are from models that include extinction and photometric shifts. The results in the lower right are from the application of models that include extinction and the photometric shifts to an independent set of standard stars. In the upper left of each panel we provide the fractional shift between the two temperatures and scatter from the 1:1 line. 


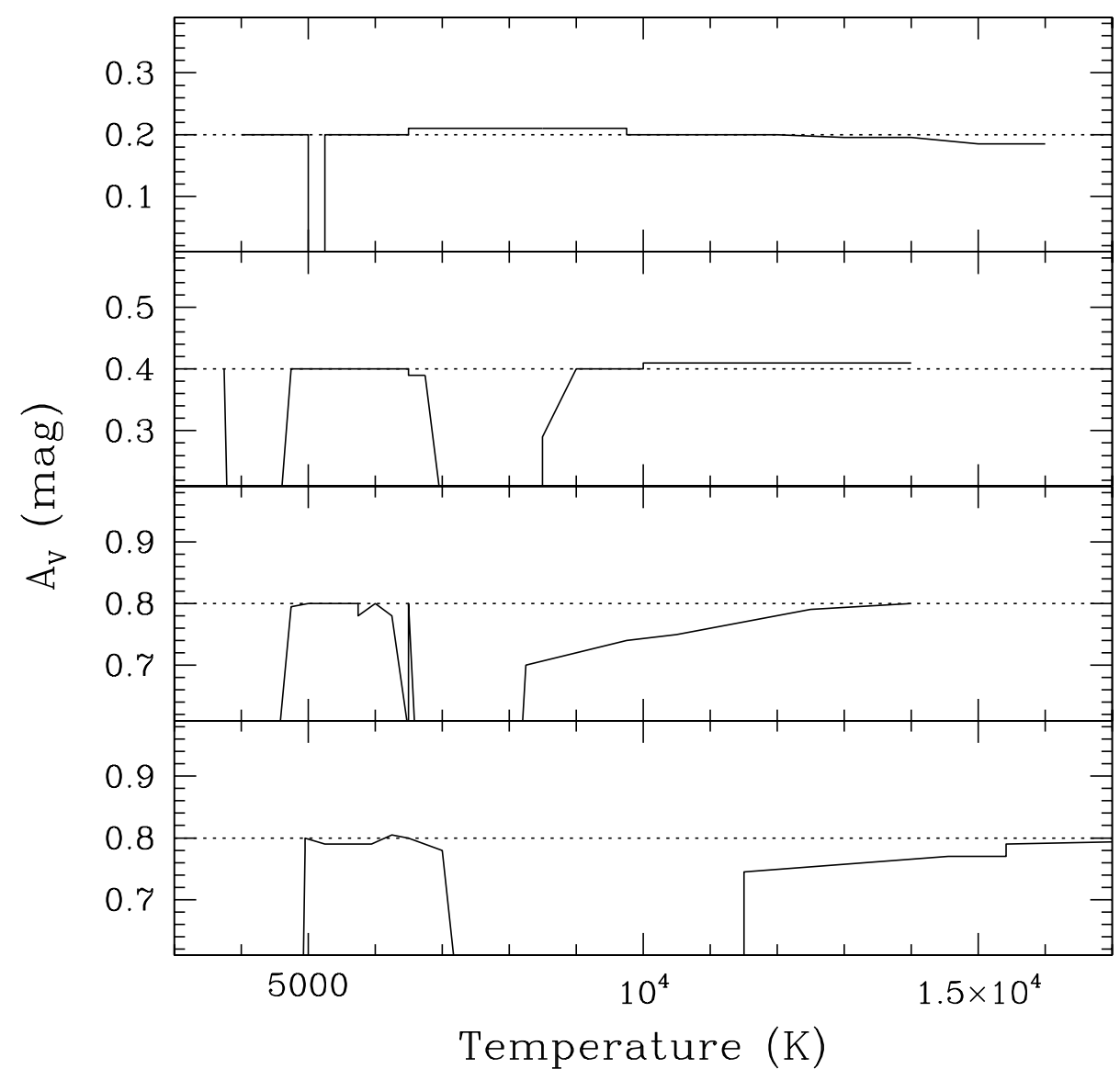

Fig. 3.- Recovering a supplemental, artificial line-of-sight extinction. The top three panels contain the recovered extinction $\left(A_{V}\right)$ as a function of recovered effective temperature (a running median in $T_{E}$ for 11 stars) drawn as a solid line. To the existing data, we added a visual extinction of 0.2 mag to generate the upper panel, 0.4 for the second panel, 0.8 for the third panel. Each panel has a dotted line that corresponds to the level of supplemental, artificial extinction. The lowest panel shows the extinction recovered from the simulation with a supplemental extinction of 0.8 (same as the third panel) but the data are plotted versus spectroscopic $T_{E}$. 


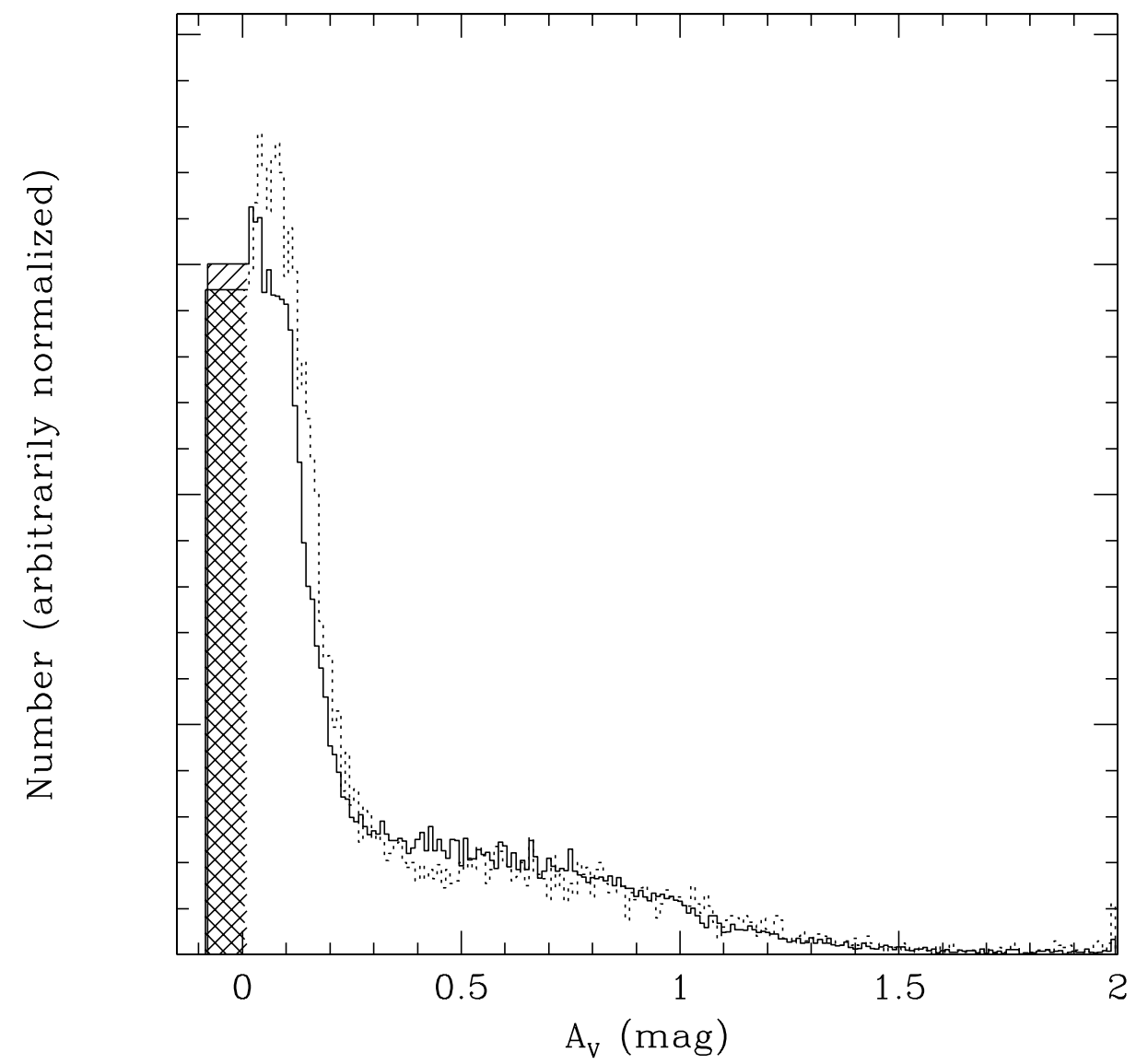

Fig. 4. - The distribution of visual extinctions for the original sample of low $T_{E}$ stars (solid line) and for the corresponding sample with larger photometric errors $\left(0.2<\sigma_{U}<0.4\right.$; dotted line). The hashed areas represent the stars for which the derived $A_{V}=0$. The two histograms have been arbitrarily normalized to match at $A_{V}=1$. The original sample is 3.4 times larger than the high $\sigma_{U}$ sample. 

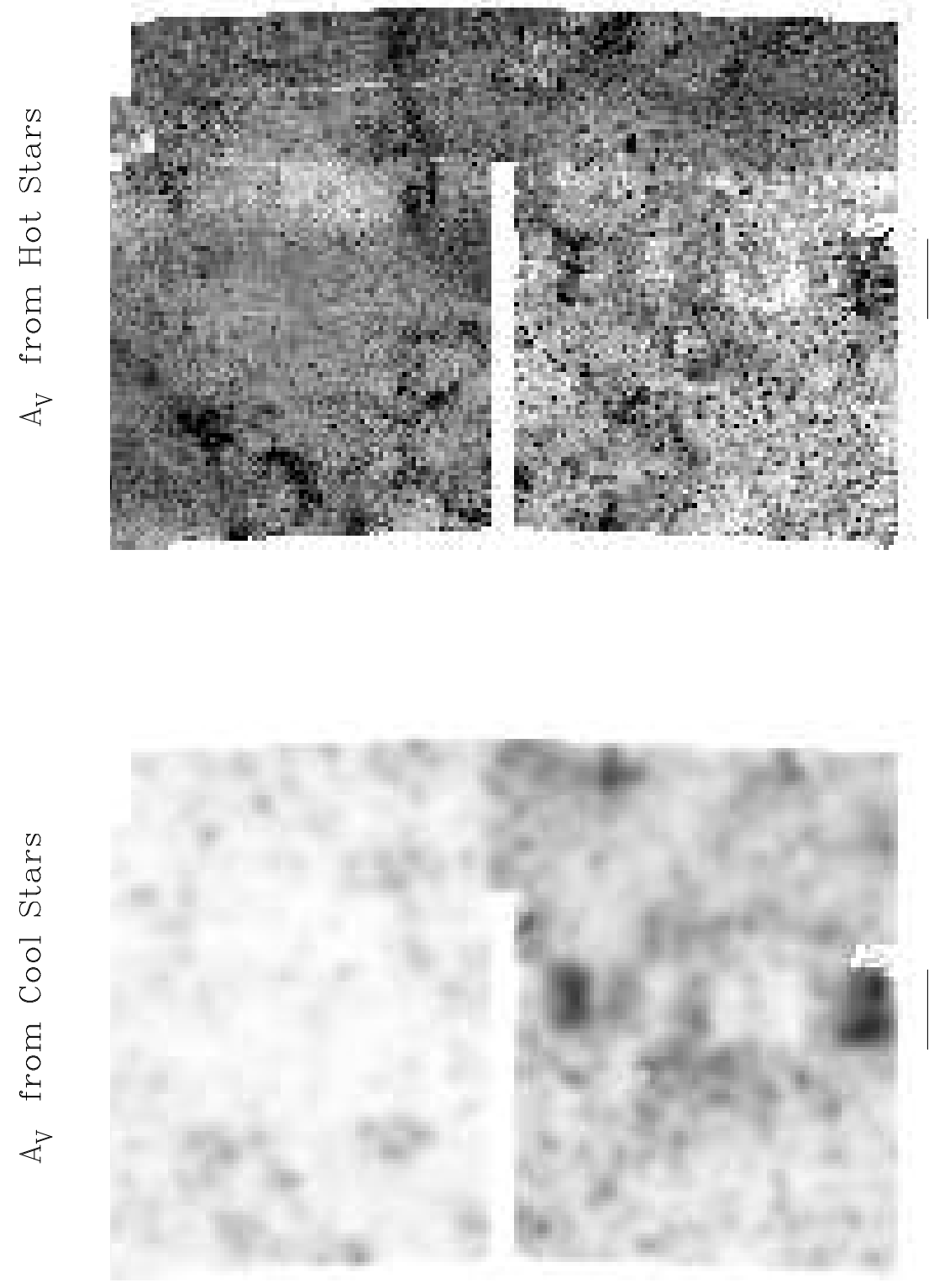

Fig. 5.- Extinction maps from stars with $12000 \mathrm{~K}<T_{E}<45000 \mathrm{~K}$ (top) and stars with $5500 \mathrm{~K}<T_{E}<$ $6500 \mathrm{~K}$ (bottom) for the region showed in Figure 1. The lower image has been smoothed with a Gaussian of 1.5 map pixel dispersion to clarify any structures. The brightness of the images are identically scaled (from $A_{V}=0$ (white) to $A_{V}=0.75$ (black)). The small vertical bar to the right of the images marks the position of a scan known to have non-photometric $I$-band data. 


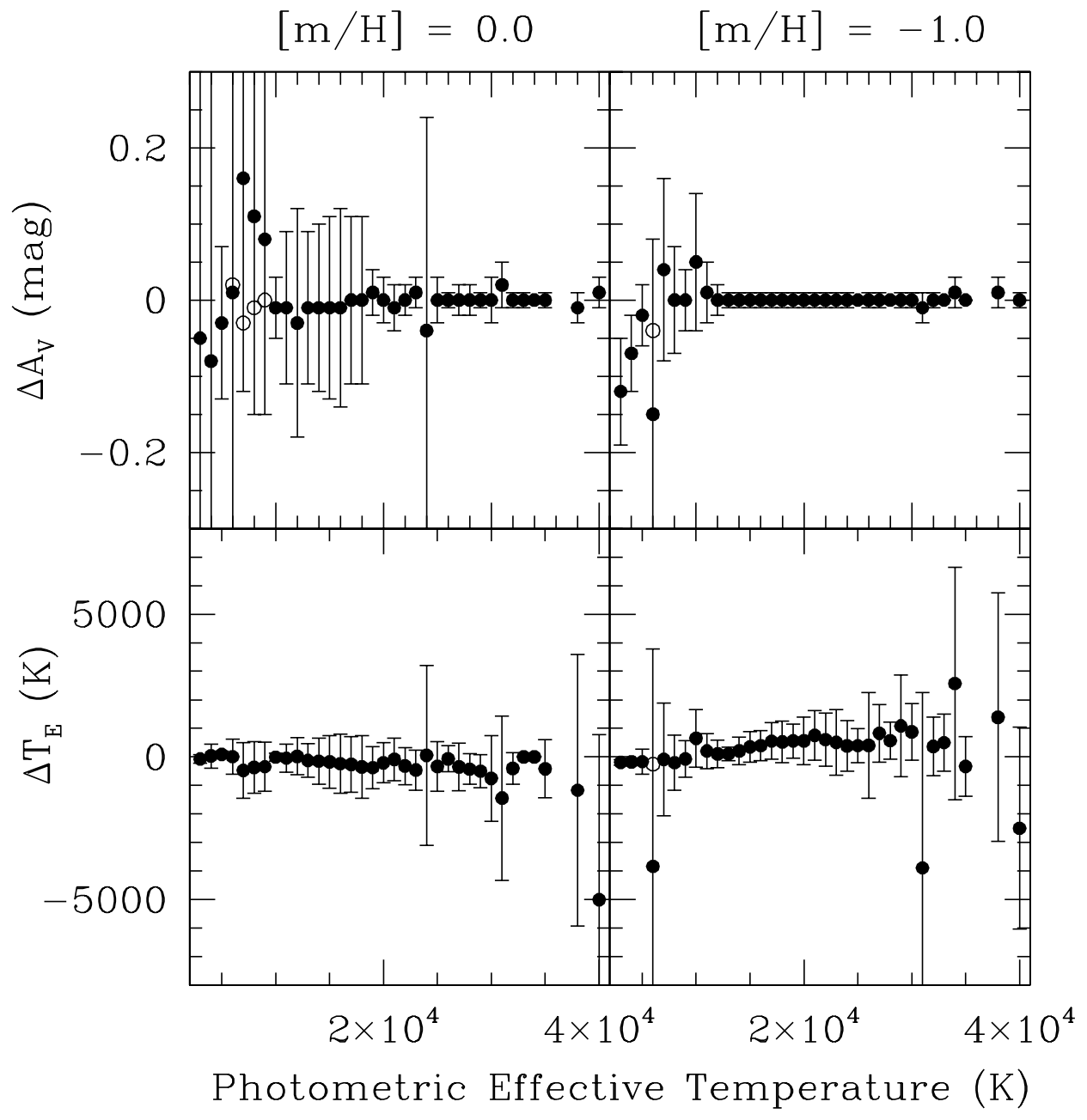

Fig. 6.- The dependence between metallicity and the derived extinction and effective temperature. The mean and dispersion of the difference between recovered quantities for various models are plotted. The comparison of results using standard models $([\mathrm{m} / \mathrm{H}]=-0.5)$ to solar metallicity models is shown in the left panels. The comparison to tenth-solar metallicity models in the right panels. The open circles at $T_{E} \sim 6000$ $\mathrm{K}$ represent the median values in those bins (see text for discussion). Errorbars are the dispersion of the mean. 


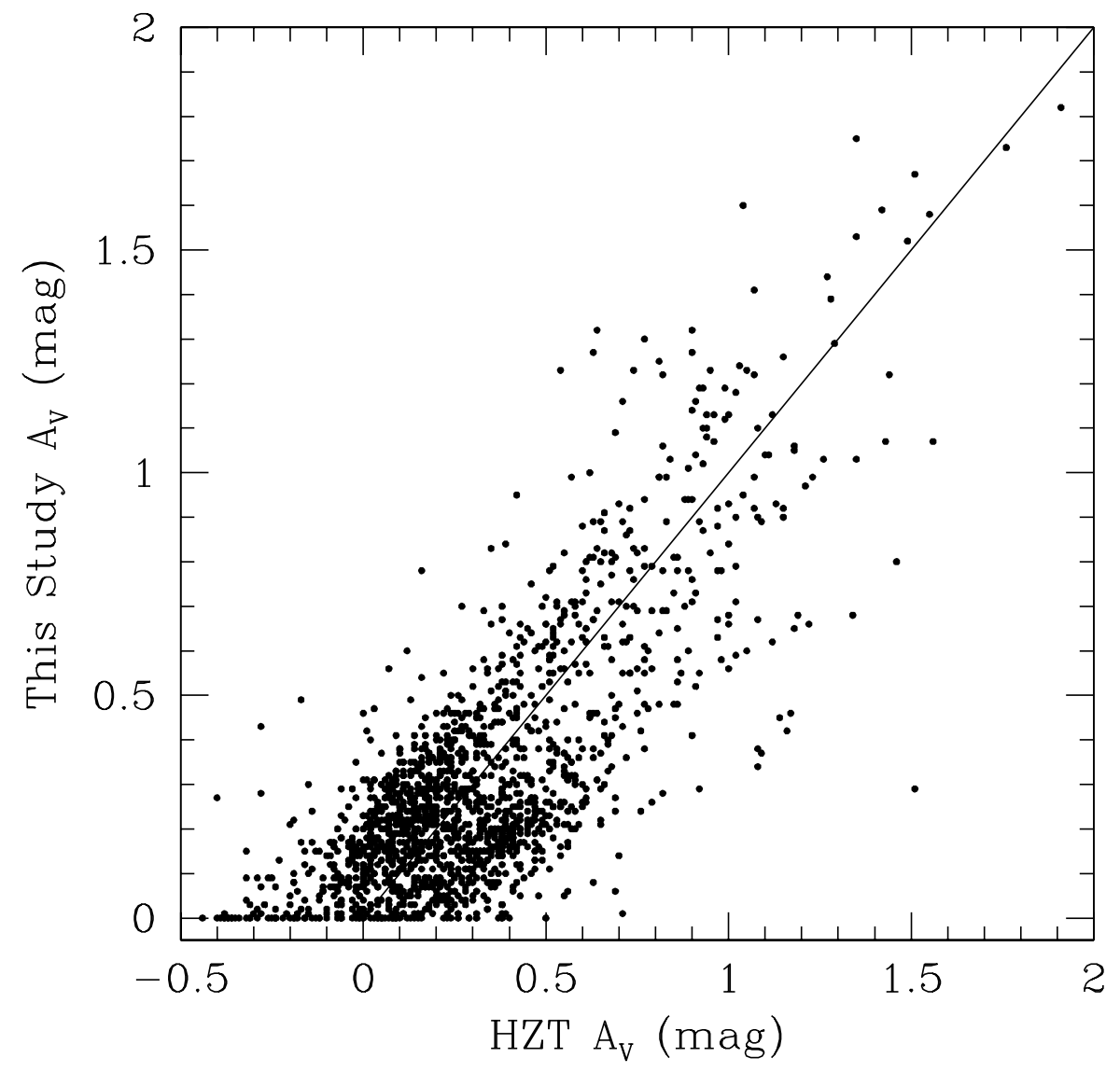

Fig. 7.- A comparison of extinction values derived for stars in common with the HZT study. We compare our values to the values they derived using the classic UBV reddening-free method. The line indicates the one-to-one correspondence and is not a fit to the data. 


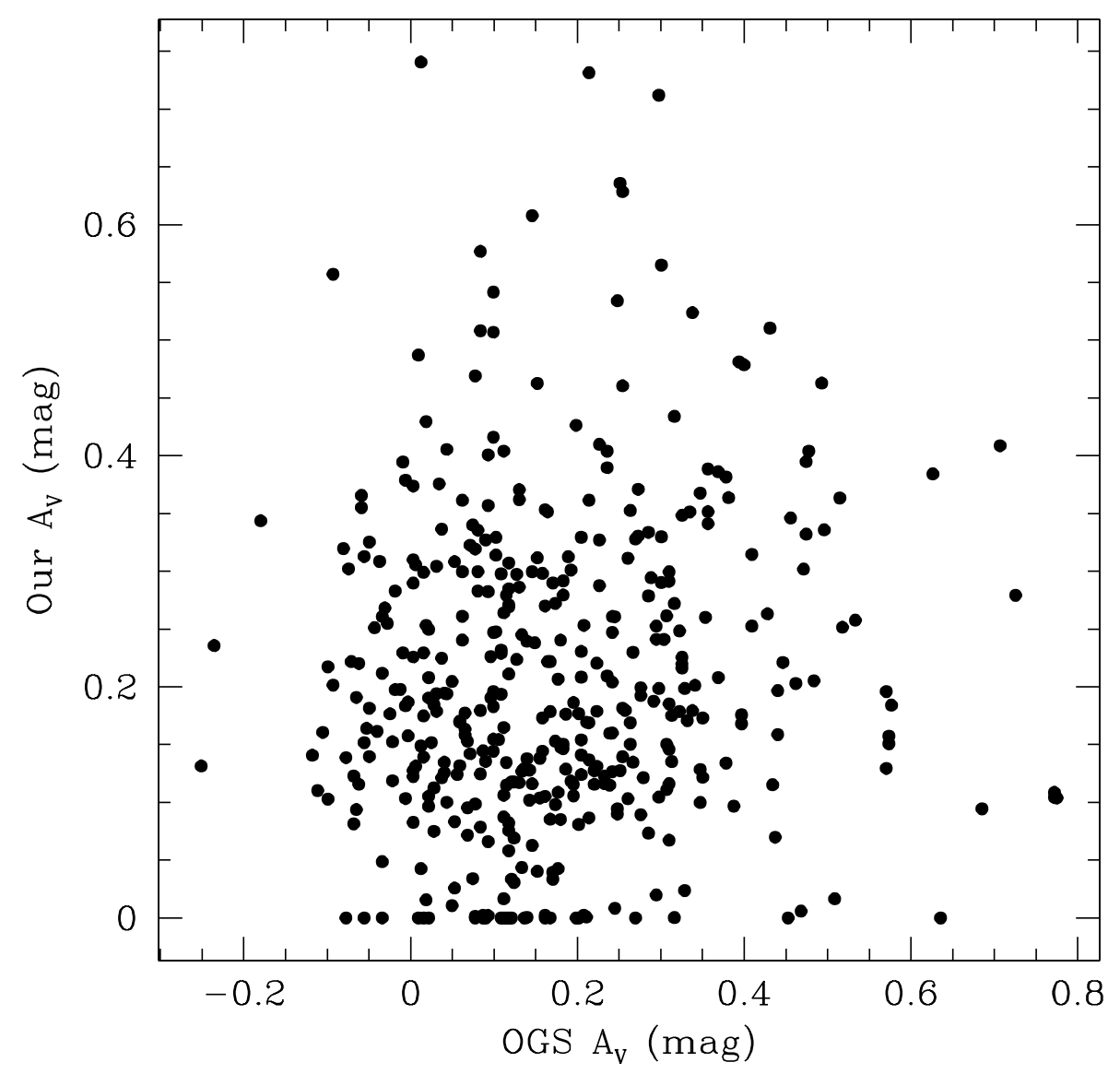

Fig. 8. - A comparison of the reddening measured by OSG to Galactic stars and the foreground reddenings implied by our map at the positions of the stars. Although the mean and dispersion of the two populations (i.e. the global statistical quantities) agree well, there is no discernible coherence in the point-by-point comparison. 


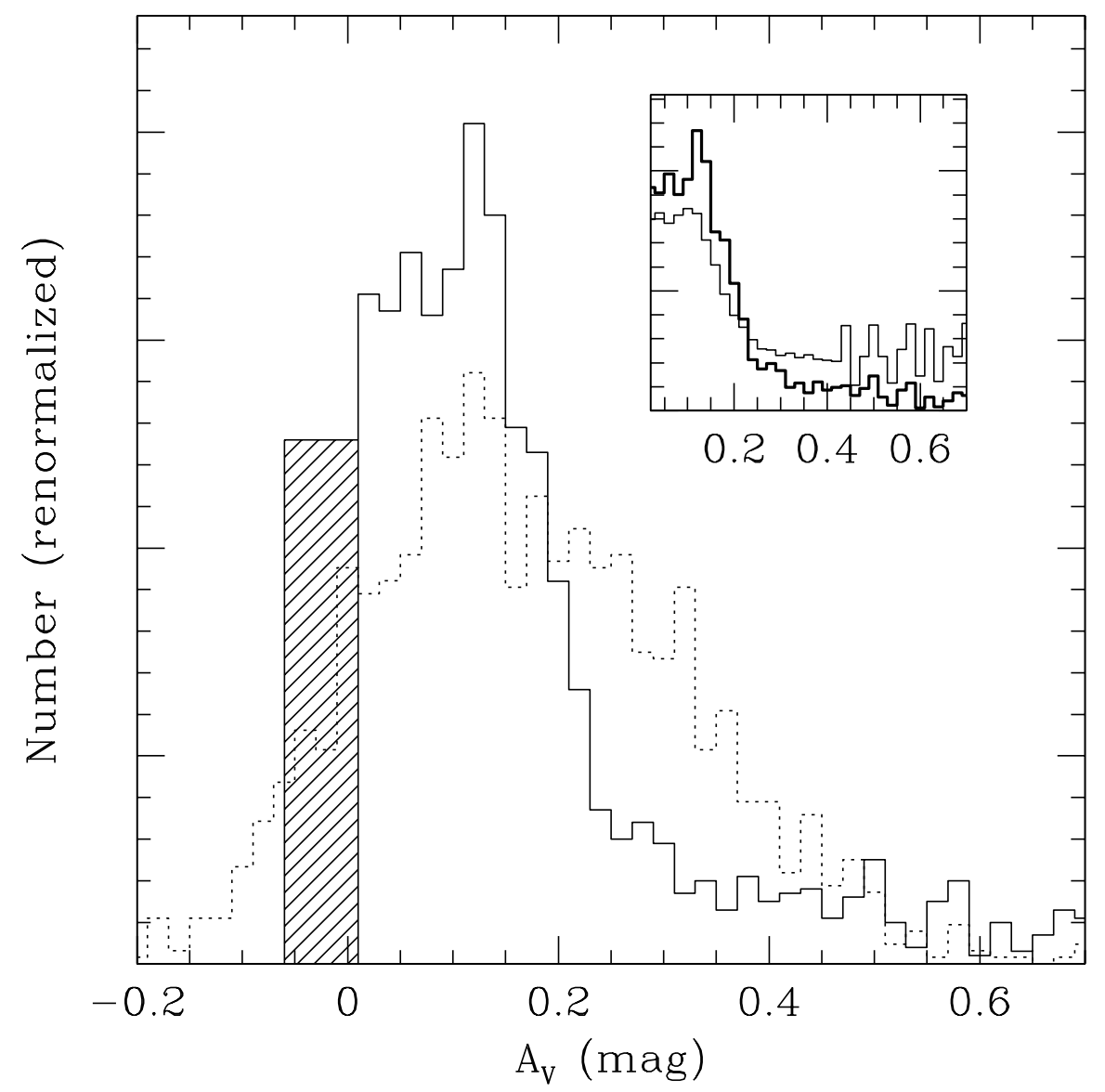

Fig. 9. - A comparison of the distribution of extinction values derived for the subset of our cool stellar population, $5500 \mathrm{~K}<T_{E}<6000 \mathrm{~K}$ (solid line) that is interpreted to be predominantly Galactic foreground stars and for Galactic foreground stars (dotted line) from OGS (1995) over their entire LMC region. The hashed box represents the number of stars with $A_{V}=0$ in our analysis $\left(A_{V}<0\right.$ is not allowed in our models). The histograms are arbitrarily normalized. In the inset we present the $A_{V}$ distribution for both our Galactic foreground stars (thick line) and LMC population (thin line). 


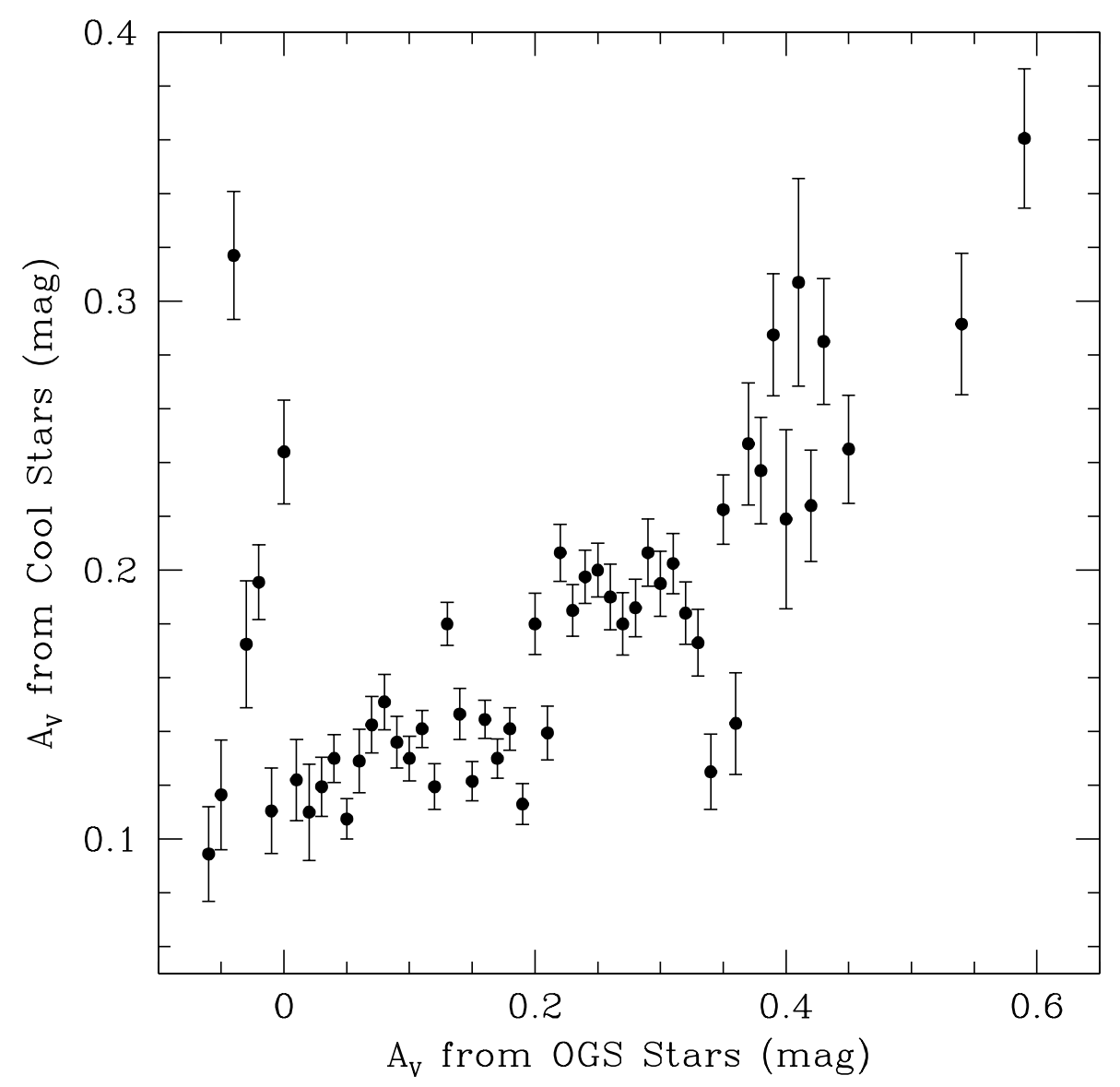

Fig. 10.- A comparison of the foreground reddenings determined from a map constructed from the OSG data and our extinction map constructed from all of the cool stars (LMC and Galactic). Pixel values are binned according to the OGS extinction value and averages of the pixels from our map are plotted (errorbars are the dispersions of the mean). The correlation is evident, indicating that structures seen in the Galactic foreground map are also evident in the LMC data, despite that lack of an apparent correlation in the extinctions of individual stars (Figure 7). 

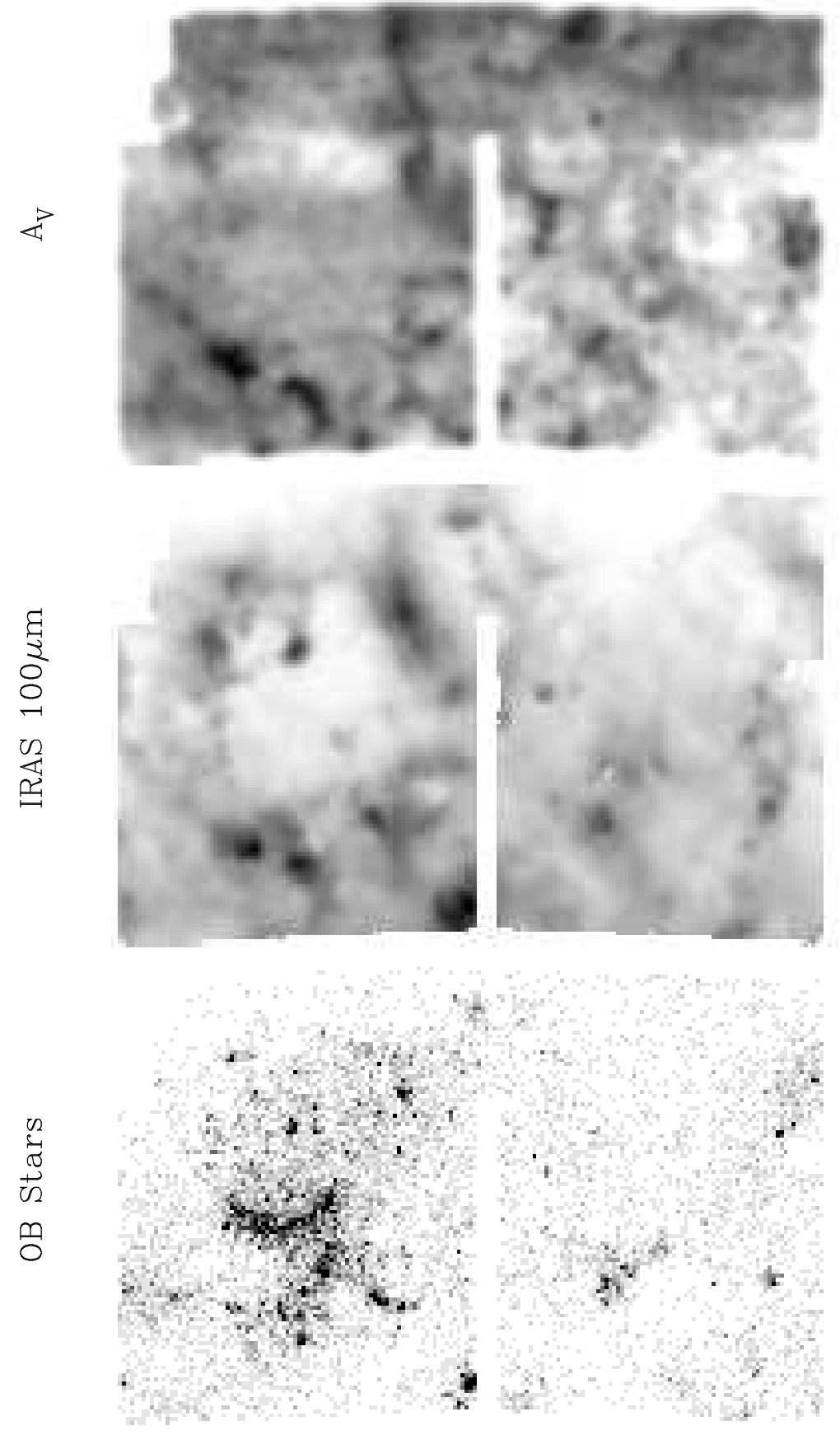

Fig. 11. - A comparison of the $A_{V}$ map derived from high $T_{E}$ stars (smoothed to match the IRAS spatial resolution), an IRAS $100 \mu \mathrm{m}$ image (log scaled), and a stellar density image of main sequence stars with $V<16.5$ and $B-V<0.5$. The vertical bar at the right of the upper panel indicates the scan that has non-photometric $I$-band data. 


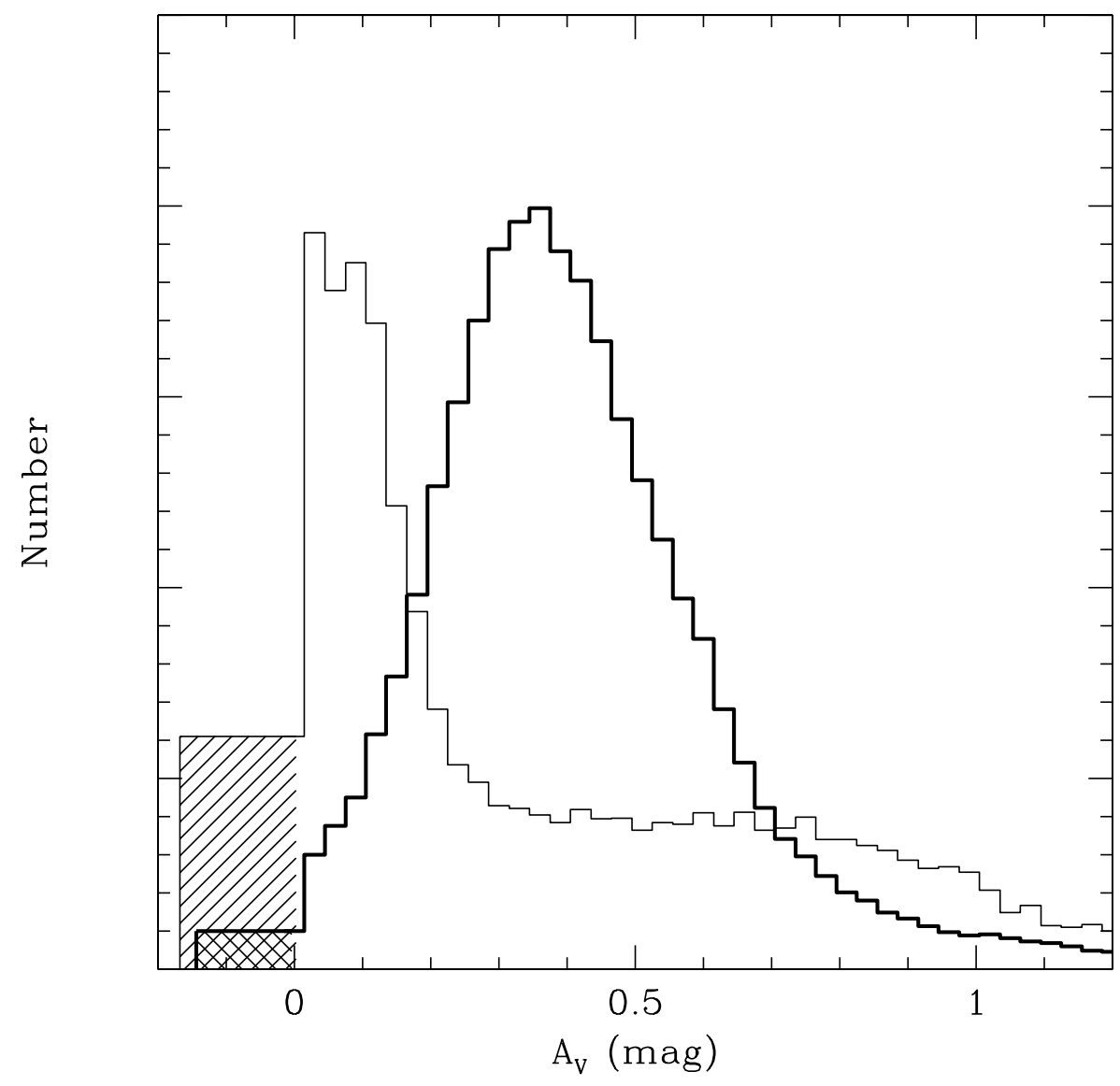

Fig. 12.- A comparison of the distribution of $A_{V}$ 's derived for the hot $\left(T_{E}>12000 \mathrm{~K}\right.$; thick solid line) and cool $\left(5500<T_{E}<6500 \mathrm{~K}\right.$; thin solid line) stars. The dashed areas at $A_{V}<0$ indicate the number of stars with $A_{V}=0$, where we have set the height of the bin equal to the number of stars with $A_{V}=0.01$ and the area to correctly represent the number of stars. The two histograms have been arbitrarily normalized. 

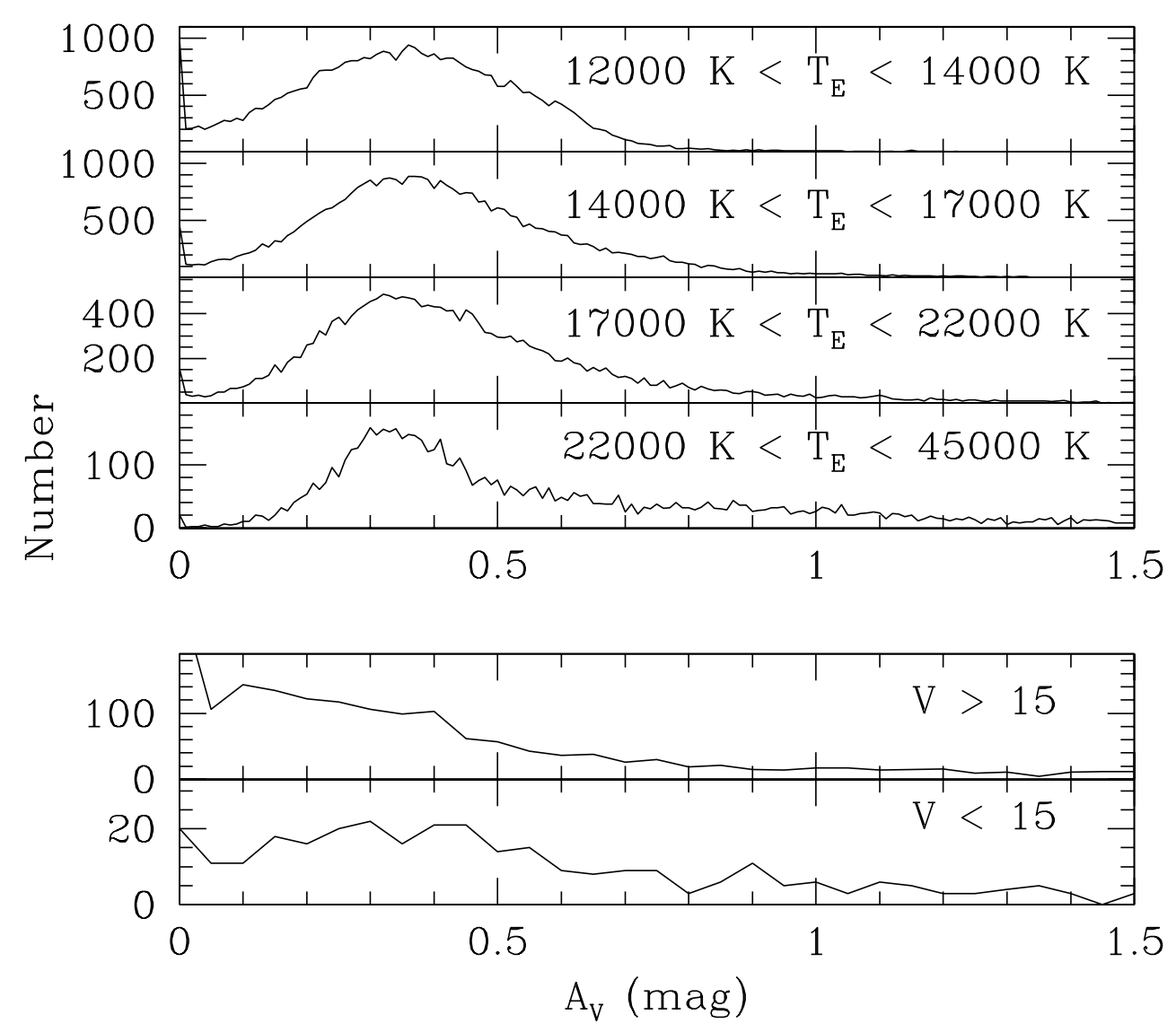

Fig. 13. - Comparison of extinction values for subsets of the high $T_{E}$ population. The lower two panels compare the values obtained by HZT using the reddening-free method. 


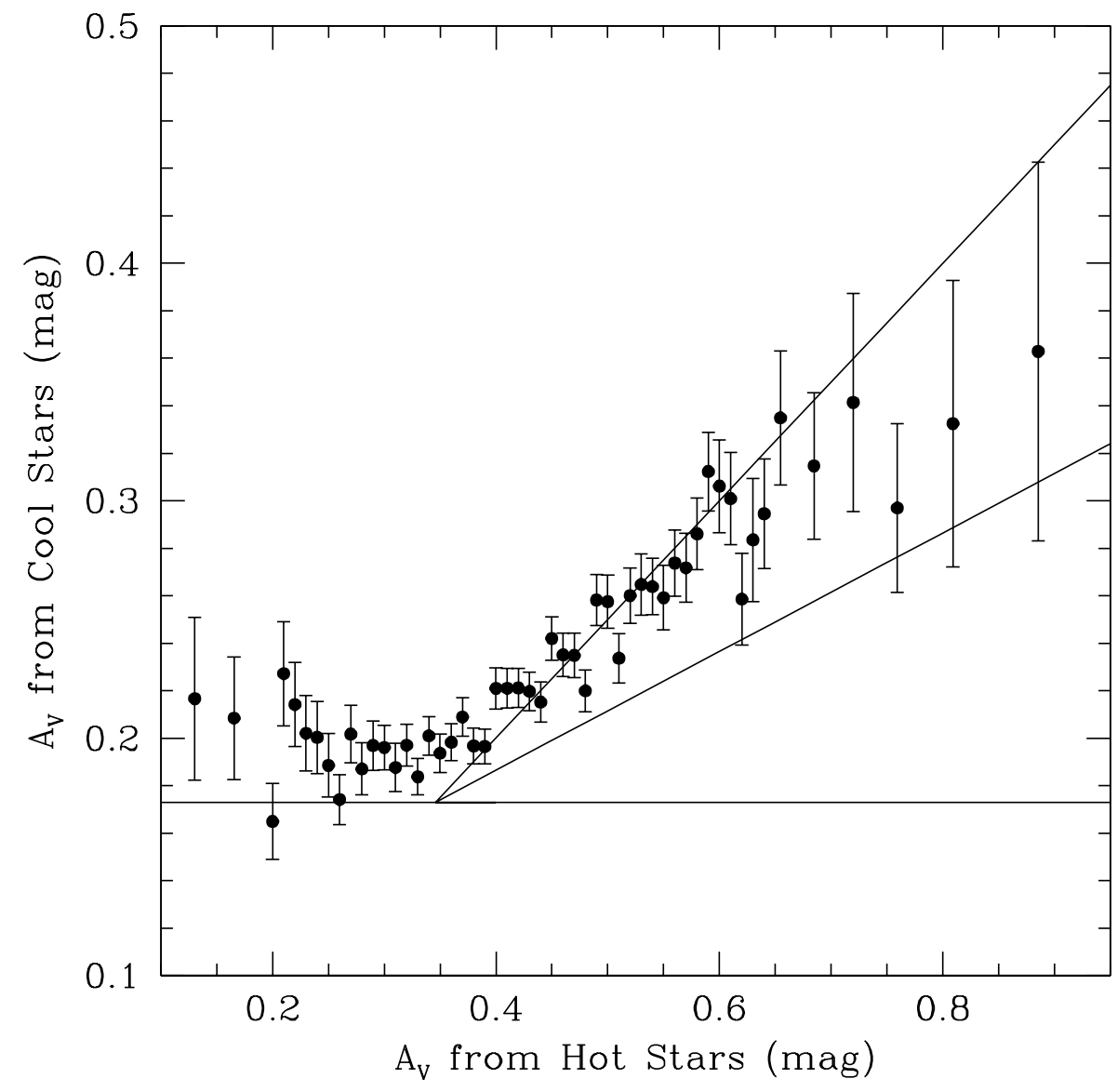

Fig. 14. - A comparison of the extinction derived for LMC stars with $12000 \mathrm{~K}<T_{E}<45000 \mathrm{~K}$ and stars with $5500 \mathrm{~K}<T_{E}<6500 \mathrm{~K}$. The data are map pixel values binned so that there are at least 200 pixels per bin. Errorbars represent standard deviation of the mean. The lines represent models as described in text. 